\title{
1 Mechanosensory stimulation via Nanchung expressing neurons can induce 2 daytime sleep in Drosophila
}

13 Shahnaz Rahman Lone ${ }^{1,2}$, Sheetal Potdar ${ }^{3}$, Archana Venkataraman ${ }^{1}$, Vasu Sheeba ${ }^{3,4 *}$ and Vijay 14 Kumar Sharma ${ }^{1 \#}$

Mechanosensory cues promote daytime sleep in Drosophila

(1)

1. Chronobiology Laboratory, Evolutionary and Integrative Biology Unit, Jawaharlal Nehru Centre for Advanced Scientific Research, P. O. Box 6436, Jakkur, Bangalore 560 064, Karnataka, India.

2. Current Address: Department of Zoology, Central University of Punjab, Bathinda, 151001, Punjab, India.

3. Behavioural Neurogenetics Laboratory, Evolutionary and Integrative Biology Unit, Jawaharlal Nehru Centre for Advanced Scientific Research, P. O. Box 6436, Jakkur, Bangalore 560064 , Karnataka, India.

4. Behavioural Neurogenetics Laboratory, Neuroscience Unit, Jawaharlal Nehru Centre for Advanced Scientific Research, P. O. Box 6436, Jakkur, Bangalore 560 064, Karnataka, India.

\# Posthumous

Running title: Mechanosensory stimulation promotes sleep.

*Co-Corresponding authors

sheeba@jncasr.ac.in; Phone: +91-080-22082987;

lonesr@gmail.com; Phone: +91-164-2864155 


\section{Abstract:}

32 The neurogenetic bases of sleep, a phenomenon considered crucial for well-being of organisms has 33 recently been under investigation using the model organism Drosophila melanogaster. Although

34 sleep is a state where sensory threshold for arousal is enhanced, it is known that certain kinds of 35 repetitive sensory stimuli such as rocking, can in fact promote sleep in humans. Here we report that 36 orbital motion aided mechanosensory stimulation promotes sleep in Drosophila, independent of the 37 circadian clock, but controlled by the homeostatic system. Mechanosensory receptor nanchung (Nan) 38 expressing neurons in the chordotonal organs mediate this sleep induction - flies where these neurons 39 are either silenced or ablated display significantly reduced sleep induction upon mechanosensory 40 stimulation. Transient activation of the Nan-expressing neurons also enhances sleep levels 41 confirming the role of these neurons in sleep induction. Thus, we show for the first time that 42 mechanosensory stimulation promotes sleep even in flies D. melanogaster and that it is mediated by 43 proprioceptors.

44 Key words: Sleep; Orbital motion; Mechanosensation; Circadian; Homeostasis; Chordotonal organs; 45 nanchung.

\section{Introduction}

48 The phenomenon of sleep is exhibited by organisms across the animal kingdom although our 49 understanding regarding functions of sleep still remains inadequate (Saper et al., 2010; Shaw et al., 50 2013). Animals shut down many vital behaviours such as feeding and reproduction and are more 51 vulnerable to predators during sleep, yet, the sleeping state constitutes a half to one third of their 52 lifespan (Allada and Siegel, 2008; Siegel, 2009; Shaw et al., 2013). It is believed that circadian 53 clocks determine the timing of sleep, while its quality (intensity) and quantity (duration) is determined 54 by the homeostatic system (reviewed in Shaw et al., 2013). Studies on organisms including fruit flies, 55 worms, zebrafish and other model organisms have revealed that many aspects of sleep are genetically 56 determined (Allada and Siegel, 2008; Crocker and Sehgal, 2010; Shaw et al., 2013). However, there

57 is also evidence to suggest that environmental stimuli like light, temperature, social cues etc., can 
58 modulate quality and quantity of sleep in flies (Lamaze et al., 2017; Parisky et al., 2016; Lone et al.,

59 2016; Lone et al., 2012; Donlea et al., 2009; Fritzgerald et al., 2006).

60 A relatively simple nervous system and ease of genetic manipulations has led to the vinegar

61 fly Drosophila melanogaster becoming increasingly preferred in the quest to unravel neuronal circuits

62 underlying many behaviours including sleep (Venken et al., 2011; Goodwin et al., 2009; Simpson,

63 2009). Over last two decades, we have begun to understand the role of circadian circuitry in

64 modulating sleep and to appreciate the contributions of homeostatic processes and their interaction

65 with the circadian circuitry (Parisky et al., 2008; Yadlapalli et al., 2018; Guo et al., 2016; Shang et al.,

66 2013; Shang et al., 2008; Sheeba et al., 2008; Liu et al., 2016; Donlea et al., 2011). Neuropeptides

67 such as Pigment Dispersing factor (PDF), small-neuropeptide factor (s-NPF), diuretic hormone 31

68 (DH31) and others serve as critical contributors to sleep properties (Kunst et al., 2014; Shang et al.,

69 2013; Sheeba et al., 2008; Shang et al., 2008; Parisky et al., 2008; Lamaze et al., 2018). Thus, we are

70 systematically unraveling the neuronal circuitry that modulates sleep using D. melanogaster (Potdar

71 and Sheeba, 2013).

72 Sleep is also modulated by age, sex, diet and several environmental conditions in flies (Keene

73 et al., 2010; Linford et al., 2012). In humans, walking during daytime has been shown to influence

74 sleep duration and quality of nighttime sleep (Morita et al., 2011). In addition to the conventional

75 prescription of somnolence-inducing drugs, non-pharmacological aids using cognitive behavioural

76 therapy, which includes, relaxation techniques that can reduce hyperarousal in patients, have been

77 used to treat insomnia (Siebern et al., 2012). Additionally, transcranial magnetic stimulation (TMS)

78 (Massimini et al., 2007), transcranial direct current stimulation (tDCS) (Marshall et al., 2006; Reato et

79 al., 2013), open-loop audio-visual stimulation (AVS) (Tang et al., 2016), acoustic stimulation (Bellesi

80 et al., 2014) have been shown to aid sleep in mammals. Although it is common knowledge that

81 babies in cradles, adults in rocking chairs and passengers in moving vehicles, fall asleep readily, yet,

82 the mechanisms underlying motion-induced sleep remain unclear. Human subjects maintained in a

83 linearly accelerated swing made a faster transition to sleep, exhibiting increased number of rapid eye

84 movements as compared to those who stayed stationary (Woodward et al., 1990). A more recent

85 study which examined daytime sleep (afternoon nap) in human subjects demonstrated that gentle 
86 rocking movements enabled participants to quickly transition from waking to sleep and enabled them

87 to experience longer duration non-rapid eye movement - NREM-stage 2 sleep (Bayer et al., 2011).

88 Yet another study on human volunteers (Perrault et al., 2019) showed that sleep latency is reduced by

89 nighttime rocking and there are fewer arousals compared to the night when volunteers were not

90 subjected to rocking. Increase in slow oscillations associated with consolidated sleep and memory

91 formation was also reported by the study (Perrault et al., 2019). Similarly, the stimulation of

92 vestibular and proprioceptive sensory inputs of mice were also seen to elicit a sustained boosting of

93 slow-wave oscillations and increased density of sleep spindles, both of which are indicators of deep

94 sleep (Kompotis et al., 2019; Bayer et al., 2011). The authors hypothesized that rocking mediated

95 sensory inputs may directly or indirectly impact sleep centers in the brain, however, the underlying

96 neuronal mechanisms remain elusive. Here we provide evidence for an ancient origin for such

97 mechanosensory stimulation induced sleep by demonstrating its existence and underlying pathways in

98 fruit flies D. melanogaster. We show that this sleep induction is reversible and independent of the

99 circadian clock but regulated by homeostatic mechanisms and mediated by the mechanosensory

100 receptor nanchung (Nan) expressing neurons located in the chordotonal organs.

101 Results: Orbital motion promotes sleep across multiple fly strains: Based on previous

102 studies of mammals including humans where rocking motion was found to induce daytime sleep

103 (Kompotis et al., 2019; Perrault et al., 2019; Bayer et al., 2011) we set out to examine the effect of

104 sustained orbital motion on flies. Here we report for the first time that gentle orbital motion (Figure

105 1a) induces sleep in D. melanogaster. Female Canton-S (CS) flies showed higher levels of sleep,

106 when provided $\underline{\text { Orbital }}$ Motion either during ㅁaytime (OMD), or continuously throughout day and

107 night (OMC) compared to controls which were not exposed to orbital motion but remained in the

108 same incubator (Figure 1b-d). In males also, OMD caused a significantly higher daytime sleep as

109 compared to controls ( $p<0.0001$; data not shown). Daytime sleep of females is generally significantly

110 lower than males in many fly strains (Huber et al., 2004; Andretic and Shaw, 2005), consequently,

111 daytime sleep induction by orbital motion is more conspicuous and consistent. Therefore, for the

112 most part, we report results on the phenomenon of orbital motion-aided enhancement of daytime sleep

113 in females, unless specified otherwise. Orbital motion of - 80-rpm, 120-rpm showed significant 
114 increase (80-rpm: $p<0.05 ; 120$-rpm $p<0.01$ ), however 600-rpm could not produce significant impact

115 on sleep increase $(p=0.21$; data not shown). Speeds below $80 \mathrm{rpm}$ were not technically feasible. We

116 found that seven different positions on the shaker platform are equally capable of sleep induction

117 ( $p>0.05$ data not shown).

118 To uncover any possible strain-specific effect, we examined four different 'wild-type' strains

119 of D. melanogaster-CS, Oregon-R $(O R), w^{1118}$, yellow white (yw) and found they also show increase

120 in daytime sleep due to OMD compared to controls $(p<0.0005$; Figure 2a-e). We verified that the

121 orbital motion induced sleep is not due to a general decline in activity since OMD flies showed

122 similar levels of activity per waking min (daytime) as controls ( $p>0.05$; Figure $2 \mathrm{f}$ ). We also observed

123 that orbital motion during daytime induces flies to fall asleep quickly after lights-on, with a

124 statistically significant decrease in daytime sleep latency for most of the strains $(p<0.0005$ for $O R$ and

$125 y w ; p<0.02$ for $C S$; for $w^{1118} p=0.16$; Figure $2 \mathrm{~g}$ ) while nighttime sleep latency was unaffected (Figure

126 2h). Thus, we detect a robust phenotype of daytime sleepiness due to OMD that is over and above

127 base line sleep across four fly strains.

128 Orbital motion mediated quiescence is "true" sleep with properties of reversibility, homeostatic

129 control and increased arousal threshold: It is possible that the quiescence induced in flies

130 experiencing orbital motion is not true sleep and merely inactivity due to inability to locomote while

131 experiencing such motion. Therefore, we examined whether OMD induced inactivity meets the

132 accepted criteria for sleep in flies, firstly its reversibility, secondly its homeostatic control and thirdly

133 the requirement for a higher arousal threshold (Campbell and Tobler, 1984; Hendricks, 2000; Shaw et

134 al., 2000). To demonstrate reversibility of OMD-induced sleep, both OMD and control groups of

135 flies were subjected to manual physical disturbance by shaking monitors for few seconds during the

136 middle of the day, which is also the peak of daytime sleep in flies, i.e. at Zeitgeber Time 06 (ZT06; by

137 convention ZT00 is lights-ON under a 12:12-h light:dark (LD) cycle) (Figure 3a). Physical

138 disturbance awoke flies in both groups $(\sim 52 \%$ and $\sim 60 \%)$ suggesting that this quiescence is

139 reversible. Importantly, flies that experienced OMD fell asleep sooner after physical perturbation as

140 compared to controls (shorter sleep latency $p<0.05$; Figure 3a,b) suggesting that OMD induced sleep

141 drive has a potent effect on flies even after a recent experience of physical disturbance. It also 
142 suggests that orbital motion is effective in promoting sleep at times other than ZT00. We also tested

143 this directly by initiating OMD at ZT04 instead of at ZT00 and similar results were observed for sleep

144 levels and latency $(p<0.001$; Data not shown).

145 We tested whether orbital motion can induce sleep in absence of light by first subjecting flies

146 to OMD under LD 12:12 for 4 days following which they were placed in constant darkness and flies

147 continue to receive OMD for $12 \mathrm{~h}$ of subjective day. We found that OMD treated flies exhibited

148 higher sleep levels even during 'subjective daytime' (Figure 3c, d, pre-pulse sleep). When exposed to

149 a brief (10-sec) light pulse of 500-lux, 4h after onset of OMD (Circadian Time 04 or CT04) (Figure

$1503 \mathrm{c}$, arrowhead), similar fraction of OMD flies awoke as controls (Figure 3c,d) (54\% of controls and

$15160 \%$ of OMD flies), which further confirmed that orbital motion-induced quiescence is reversible.

152 Prior to light pulse, OMD flies exhibited significantly higher daytime sleep as compared to controls ( $p$

$153<0.05$; Figure $3 \mathrm{c}, \mathrm{d}$ ), however, following light pulse, this difference was not statistically significant

154 ( $p=0.22$; Figure $3 \mathrm{~d}$ ). This may be due to overall reduction in sleep for both controls and treated flies

155 brought about by the pulse of light probably due to impact on circadian and sleep parameters.

156 Like humans, flies are also susceptible to the arousal promoting effects of caffeine (Shaw et

157 al., 2000). We asked whether the OMD is reversible by caffeine. Out of three groups of flies, two

158 received OMD while the third group served as control. Both sets of OMD exposed flies slept

159 significantly more than controls ( $p<0.05$; Figure 3e-left panel) on the day prior to caffeine treatment

160 (pre-caf). On the next day, immediately after lights-ON, one of the OMD exposed groups was fed

161 caffeinated food (1-mg/ml, OMD-caf), while the other two groups (OMD and controls) were

162 transferred to fresh food medium without caffeine (Figure 3e-right panel). Flies which received

163 caffeine along with OMD, slept significantly less during daytime as compared to those exposed only

164 to OMD $(p<0.0005)$ but not less than the controls $(p=0.98)$ (Figure 3f). This suggests that orbital

165 motion-induced sleep can be blocked by pharmacological intervention, emphasizing reversibility of

166 sleep induced by orbital motion and that inactivity is not due to the orbital motion preventing

167 locomotion of flies. Expectedly, nighttime sleep of flies fed with caffeine during the day, was also

168 lower than the other groups possibly due to the persistence of arousal-promoting effect of caffeine

169 into the night. 
170 To examine whether orbital motion-induced sleep can be modulated by homeostatic pathways

171 we divided flies into three groups, the first group was subjected to orbital motion for $12 \mathrm{~h}$ during

172 daytime (ZT00-ZT12, OMD), the second group only during the first half of daytime (ZT00-ZT06,

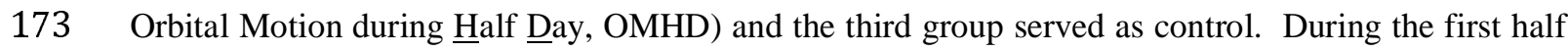

174 of the day there was a statistically significant increase in sleep in both groups exposed to orbital

175 motion (OMD and OMHD; $p<0.0005$; Figure 3g,h) as compared to controls. However, during the

176 second half of the day, OMHD group of flies slept significantly lesser than OMD and even lower as

177 compared to controls $(p<0.0005$; Figure $3 \mathrm{~g}, \mathrm{~h})$. This is suggestive of a 'negative sleep rebound'

178 during the second half of the day, possibly due to accelerated decline in sleep drive that is believed to

179 occur via homeostatic processes (Hendricks et al., 2000; Shaw et al., 2000). Taken together, we

180 demonstrate that most of the signature features of sleep are conserved in flies exposed to OMD

181 suggesting that OMD-mediated quiescence is in fact "true" sleep.

182 Orbital motion induced sleep is independent of circadian clock genes period and Clock-: Circadian

183 clocks are known to influence the timing of sleep (Borbely, 1982; Borbely et al., 2016). Moreover,

184 we saw that the behaviour of OMD induced sleep persists even under constant darkness during

185 subjective day (Figure 3c), so we asked if the circadian clock is required for this sleep-induction. We

186 subjected flies carrying loss-of-function mutation for the period $\left(\right.$ per $\left.^{0}\right)$ and $\operatorname{clock}\left(C l k^{\mathrm{Jk}}\right)$ genes to

187 OMD, and found that flies exposed to orbital motion showed a statistically significant increase in

188 daytime sleep ( $p<0.001$ for per $^{0}$ and $p<0.0005$ for $c l k^{\mathrm{jrk}}$; Figure $4 \mathrm{~b}$ ) as compared to controls, while

189 their activity counts per waking min did not differ. This suggests that core circadian clock genes

190 period and Clock are not necessary for OMD-induced sleep.

191 Mechanosensory signals via Nanchung expressing neurons mediate orbital motion aided sleep

192 induction independent of other sensory modalities such as vision and olfaction: The

193 mechanosensory system helps organisms to integrate mechanical information to guide their locomotor

194 activity (Tuthill and Wilson, 2016) and locomotion is inhibited during sleep. To examine how orbital

195 motion may transduce signals to the sleep circuit, we examined various mechanosensory signaling

196 pathways. Fruit flies D. melanogaster have the ability to sense and respond to touch via specialized

197 mechanosensory centers, the chordotonal organs (Kim et al., 2003), which have been also shown to 
198 relay temperature and vibration signals to circadian clocks via yet unknown mechanisms (Sehadova et 199 al., 2009; Simoni et al., 2014). Additionally, mechanosensory neurons present on the legs help flies to 200 avoid aversive conditions by inducing walking behaviour (Ramdya et al., 2014). The chordotonal 201 organs express mechanoreceptor Nanchung (Nan), which on the basis of sequence similarity is 202 predicted to be an ion channel subunit, similar to vanilloid-receptor-related (TRPV) channels and is 203 activated by forces ranging from osmotic to mechanical pressures (Kung, 2005). These channels also 204 respond to changes in gravity, sound and humidity levels (Kamikouchi, 2009).

205 We found that flies carrying loss-of-function mutation in the nan gene ( an $^{\mathrm{dy} 5}$ ) (Kim et al., 206 2003) slept similar ( $p=0.70$, Figure 5a-c-) to heterozygote $n a n^{d y 5} /+$ suggesting that reduced nan levels 207 does not hamper sleep induction by orbital motion. Since orbital motion is most likely to be 208 transduced to the fly via mechanosensory pathways we targeted the nan expressing neurons by 209 expressing the pro-apotoptic gene hid (nanGAL4>UAShid). When we compared sleep change 210 (average sleep of controls with sleep of flies receiving OMD - $\triangle$ sleep), nanGAL4>UAShid flies 211 showed a significantly reduced response to OMD as compared to their parental controls $(p<0.0005$ for 212 nanGAL4/+ and $p<0.01$ for $U A S /+$; Figure 5d-g). In a separate experiment, we blocked synaptic 213 signaling in nan-neurons using an active form of tetanus toxin (nanGAL4>UAStnt(ac)) along with 214 controls expressing an inactive form (nanGAL4>UAStnt(inac)). $\triangle$ sleep in nanGAL4>UAStnt(inac) 215 is significantly more $(p<0.01$, Figure $5 \mathrm{~h}-\mathrm{j})$ than nanGAL4 $>U A \operatorname{Stnt}(a c)$. These results suggest that 216 orbital motion induces sleep via sensory input received via Nan-neurons. We have found similar 217 results when nan expressing neurons were silenced by UASdORCKCl. Sleep is increased in 218 nanGAL4<UASdORKNC flies subjected to OMD ( $p<0.05)$, however in nanGALA<UASdORKC flies, 219 there was no significant change in sleep when flies were subjected to OMD ( $p=0.88$; data not 220 shown).

221 Since mechanosensory cues are also perceived by auditory and tactile systems we examined mutant 222 flies for the gene потрс2, an ion channel essential for mechanosensory transduction, known to have 223 defects in the receptors of tactile bristles (Walker et al., 2000) and found nompc2 flies exposed to 224 OMD slept significantly more than controls $(p<0.0001$; Figure $6 a)$, which suggests that 225 mechanosensory cues emanating from auditory and tactile organs are not involved in orbital motion 
226 mediated sleep-induction, and that mechanosensory signals from parts of the chordotonal organs other 227 than those responsible for auditory signals are likely to be involved in this sleep phenotype. Because 228 mechanosensory cells are also present in the antennae, we examined the role of mechanosensory cues received by the antennae by surgically removing both the antennae of $C S$ flies (CS ant). We found

230 that antennae-less flies also exhibit sleep induction due to OMD ( $p<0.005$ for controls and $p<0.05$ for

231 CS ant Figure 6b), which suggests that mechanosensory signals from the antennae are not necessary 232 for this sleep phenotype.

233 Orbital motion, could in principle, have some direct/indirect effect on sensory systems such 234 as olfaction, vision and audition, which in turn may affect sleep circuitry. A previous study on rats 235 has shown that olfactory stimulation can induce slow wave activity (Fontanini and Bower, 2006), 236 while cutaneous stimulation in cats resulted in synchronised brain activity akin to sleep (Pompeiano 237 and Swett, 1962). A more recent study on human subjects showed that acoustic inputs by way of 238 brief auditory tones $(0.5-4 \mathrm{~Hz})$ induced slow wave activity with features very similar to natural sleep 239 (Tononi et al., 2010). To test for such effects we blocked olfactory and visual inputs to flies while 240 they were subjected to OMD. We tested null mutants of $\operatorname{Orco}\left(\operatorname{Or} 83 b^{\circ}\right)$, which are defective in their 241 olfactory ability for most odors (Larsson et al., 2004) and found that mutant flies subjected to OMD 242 show increased $(p<0.0001$; Figure $6 c)$ daytime sleep as compared to controls. Further, when we 243 silenced the $O r 83 b$-expressing olfactory neurons by expressing potassium channels (UASkir2.1), flies 244 exposed to OMD showed increased sleep ( $p<0.0001$; Figure 6c-e) as compared to controls, which 245 suggests that olfactory signals do not play any role in mechanically stimulated sleep induction.

246 To examine the role of vision, we subjected CS flies to LD cycles for three days and then 247 transferred them to DD along with orbital motion for the first 12 hours under darkness and estimated 248 sleep levels during subjective day. We found a statistically significant increase $(p<0.05$; Figure $6 \mathrm{f}, \mathrm{h})$ 249 in daytime sleep in OMD flies compared to controls even under darkness, which suggests that visual 250 cues are not needed for sleep induction. We also found that norpA mutants, known to have defective 251 vision (Bloomquist et al., 1988) also showed sleep induction when exposed to OMD ( $p<0.0001$;

252 Figure $6 \mathrm{~g}, \mathrm{~h})$. Taken together these results suggest that orbital motion aided sleep induction can occur 
253 even under sub-optimal performance of sensory modalities such as olfaction, vision, hearing and 254 tactile sensation.

255 Transient activation of Nan-neurons induces sleep in absence of orbital motion: Since our results 256 thus far suggest that nan-neurons transduce mechanosensory stimuli produced by orbital motion to 257 sleep centers. We silenced Nan neurons transiently by over expressing UASshibire ${ }^{t s}$. At lower 258 temperature of $21^{\circ} \mathrm{C}$, sleep change ( $\triangle$ sleep) of nanGAL4 /UASshibire ${ }^{t s}$ is similar $(p=1.0$, Figure $7 \mathrm{a}$, 259 c) to controls during daytime and nighttime. When we increased temperature to $28{ }^{\circ} \mathrm{C}$, silenced flies 260 (nanGAL4 /UASshibire ${ }^{\text {ts }}$ ) do not show sleep induction compared to undisturbed controls as evident by 261 the significantly lower $\Delta$ sleep ( $p<0.0005$ for daytime and $p<0.05$ for nighttime). We asked 262 whether transient activation of Nan-neurons alone can induce sleep in the absence of orbital motion.

263 We expressed temperature gated cation channel $A T R P A 1$ under the nanGAL4 driver and found that at 264 sub-threshold temperatures $\left(21^{\circ} \mathrm{C}\right)$ nanGAL4/dTRPAl flies sleep as much as nanGAL4/+ and $265 d T R P A 1 /+$ controls $(p>0.05$; Figure $7 \mathrm{~d}, \mathrm{e})$. However, when the temperature was raised to $28{ }^{\circ} \mathrm{C}$ 266 (activation temperature of dTRPA1, Viswanath et al., 2003), and Nan-neurons are expected to 267 enhance firing frequency (Hamada et al., 2008; Tang et al., 2013), sleep change is significantly higher 268 in nanGALAIdTRPA1 flies as compared to nanGAL4/+ $(p<0.05)$ and dTRPA1/+ $(p<0.005)$ controls

269 (Figure 7d, e), which suggests that electrical activity of Nan-neurons contributes to orbital motion 270 induced sleep.

273 very high rates that could lead to paralysis which we would not be able to distinguish from sleep we 274 carried out another study using a behavioural means to perturb sleep. Male flies become hyperactive 275 in the presence of females and show reduced sleep (Fuji et al., 2007; Lone and Sharma, 2012) we 276 used this behavioural paradigm to disrupt sleep in nanGAL4/dTRPAl males, where we expect the 277 transient activation of Nan-neurons to induce sleep at $28{ }^{\circ} \mathrm{C}$. We paired males of nanGAL4/dTRPA1 278 with $C S$ females and found that at $28{ }^{\circ} \mathrm{C}$ the male-female couples show decreased sleep as compared 279 to male-male pairs, or solitary nanGAL4/dTRPAl males $(p<0.0001$; Figure $7 \mathrm{f}-\mathrm{h})$ confirming the 
280 finding that activation of Nan-neurons does not cause a paralytic effect in either males or females and

281 its effect is more likely to occur via its action on sleep circuits.

282 We fed nanGALAIdTRPA1 flies with caffeine which is known to prevent sleep and cause 283 arousal while simultaneously activating the neurons by raising the temperature to $28{ }^{\circ} \mathrm{C}$. Caffeinated 284 nanGAL4IJTRPAl flies showed significantly lower sleep as compared to flies fed with sucrose 285 (vehicle) specifically under $28{ }^{\circ} \mathrm{C}$ when nanGAL4 driven neurons are excited $(p<0.0001$; data not 286 shown). This shows that Nan neuronal activation does not lead to paralysis and that the sleep induced 287 by transient hyperexcitation is reversible.

Electrical stimulation of arousal neurons can override orbital motion-induced sleep: Previous studies in Drosophila suggest a key role for 1- $\mathrm{LN}_{\mathrm{v}} \mathrm{s}$ in sleep regulation by maintaining the arousal state

290 (Sheeba et al., 2008; Shang et al., 2008; Shang et al., 2011; Shang et al., 2013; Parisky et al., 2008;

291 Donlea, 2009). We asked whether orbital motion can induce sleep when the 1- $\mathrm{LN}_{\mathrm{v}}$ neurons are 292 activated by examining flies with c929GAL4 driving NaChBac (c929/NaChBac, Nitabach et al., 293 2006), Figure 8b,c). We found daytime sleep of $c 929 / \mathrm{NaChBac}$ is lower $(p<0.005$ for $c 929 / d O R K \Delta-$ $N C$ and $p<0.05$ for $c 929 G A L 4)$ than controls (c929/dORKA-NC and $c 929 G A L 4)$. Further, we

295 transiently activated $1-\mathrm{LN}_{\mathrm{v}} \mathrm{s}$ using dTRPAl in adults and found that at $28{ }^{\circ} \mathrm{C}$, c929/dTRPAl flies 296 showed significantly lower sleep $(p<0.001, p<0.05$ in comparison to $c 929 /+$ and $d T R P A 1 /+$; Figure 297 8d-f) as compared to parental controls. However, at sub-activation-threshold temperature of $21^{\circ} \mathrm{C}$ all 298 three (c929/dTRPA1, c929/+ and dTRPA1/+) genotypes showed a statistically significant increase in sleep when exposed to OMD, and there was no difference in sleep between the experimental and 300 control flies $(p>0.91$; Figure 8f). Thus, arousal signals due to electrical excitation of 1-LNvs can 301 override sleep signals induced by OMD.

302 Discussion: Our study examined and found that gentle-movement-induced sleep that humans 303 experience can be replicated in several fly strains and attempted to decipher the neuronal circuits 304 involved. We designed an experimental paradigm involving exposure to orbital motion (Figure 1a) 305 and found that it promotes daytime sleep in fly strains without compromising their overall locomotor 306 activity levels (Figure 2) and that this phenotype is especially evident in females which otherwise 307 exhibit significantly lower daytime sleep levels. Local field potentials in the female fly brain were 
308 previously shown to differ between daytime and nighttime sleep (van Alphen et al., 2013) suggesting 309 qualitative differences between them, with daytime sleep deemed to be lighter. Our studies are 310 consistent with previous studies that showed that rocking can induce sleep in humans and mice 311 (Perrault et al., 2019; Kompotis et al., 2019; Bayer et al., 2011). Orbital motion induced quiescence is 312 completely reversible by pharmacological and behavioural interventions (Figure 3) and thus qualifies 313 this criterion for sleep. OMD induced sleep does not require functional circadian clocks (Figure 4) 314 but appears to act via homeostatic processes (Figure 3g, h). Null mutations of core clock genes 315 continue to respond to the OMD and show consistent increase in sleep (Figure 4). We showed that 316 the orbital motion induced sleep relies on mechanosensory Nan-neurons present in the chordotonal 317 organs. The role of Nan expressing neurons is evident by the fact that mechanically stimulated flies 318 with ablated (nanGAL4/UAShid) or silenced (nanGAL4/UAStnt) Nan-neurons show a significantly 319 reduced sleep induction (Figure 5). The fact that activation of Nan-neurons alone causes sleep 320 induction in absence of orbital motion suggests that mechanosensory cues act to activate the 321 chordotonal Nan-neurons to promote sleep. Our results clarify that tactile bristles and antennae, 322 considered to be the key components of fly mechanosensory system (Kim et al., 2003; Walker et al., 323 2000; Effertz et al., 2011; Yan et al., 2013) are not involved in mechanosensory stimulation mediated 324 sleep induction (Figure 6b). Thus, we demonstrate here for the first time that mechanosensory 325 stimulation can lead to sleep induction in fruit flies D. melanogaster, and that the underlying 326 mechanisms involve activation of Nan-expressing mechanoreceptor neurons located in the 327 chordotonal organs.

328 Most sensory stimuli are perceived by G-coupled protein receptors, except touch, vibration, 329 and pressure, which are sensed by mechanosensory receptors (Kung, 2005). Mechanosensory 330 neurons respond to such cues by converting them into receptor potentials (Gong et al., 2004) and 331 many organisms have evolved the ability to respond to ligands, which changes the magnitude of such 332 receptor potentials. We believe that differences in the type of motion, and intensity of mechanical 333 stimulation would invoke widely different responses. Rapid to-and-fro motion of about 1000-rpm 334 would cause sleep deprivation (Huber et al., 2004), whereas slow orbital motion of 80-120-rpm would 335 result in sleep induction. A similar feature of no-impact followed by sleep inducing, followed by 
336 sleep disrupting effect of sensory stimulation was also hypothesized to explain the effect of increased

337 acoustic stimulation in humans (Bellesi et al., 2014). Stimulation above a certain intensity

338 threshold can effectively induce sleep while stimulation below the threshold is ineffective 339 and stimulation well past the threshold can be disruptive Since increased sensory threshold is a 340 cardinal feature of the sleeping state, the ability of repetitive low amplitude sensory input to induce 341 sleep in an awake animal is counter-intuitive. However, recent studies in mammals including humans 342 (Perrault et al., 2019; Kompotis et al., 2019; Bayer et al., 2011) showed that rocking can enhance the 343 propensity to enter into deep-sleep state. The authors hypothesize that certain types of low-grade 344 sensory stimuli can potentially increase sleep propensity by impinging on brain regions which receive 345 inputs regarding a relaxed or low-stress state or by stimulating sleep centers in the hypothalamus or 346 brain stem region. Alternatively, the authors propose that rocking enhances the degree of synchrony 347 in neural activity in thalamo-cortical regions, which in turn are associated with deep sleep (Bayer et 348 al., 2011).

349 Non-pharmacological intervention to alleviate sleep disorders are highly desirable since 350 pharmacological agents often have off-target effects and lingering effects persisting into the wakeful 351 state. The fly model for sleep has been particularly useful in unraveling genetic components 352 underlying sleep regulation as well as the neuronal pathways involved, including cellular and 353 molecular components (reviewed in Donelson and Sanyal, 2015). Our finding of a pathway of 354 sensory stimulation that can alleviate sleep levels in this model organism should enable future studies 355 that suggest efficient therapeutic measures to treat sleep defects in a wide range of conditions 356 including circadian sleep phase syndromes, neurodegenerative conditions with associated sleep loss, 357 metabolic syndrome and so on.

358 Materials and methods: Flies were collected, sexed, and maintained as virgins in a temperature and 359 humidity-controlled room $\left(25 \pm 1^{\circ} \mathrm{C}\right.$ temperature and $75 \%$ relative humidity) under 12:12-h light/dark 360 (LD) cycles, at a density of 30 flies per vial. In most of our experiments, light intensity of 500-lux 361 was used during the light phase of the LD cycles. Four-day old flies were loaded into 65mm tubes 362 with 5\% sucrose and cotton at other end by anesthetizing them using carbon-di-oxide. Flies were 
363 recorded with the help of DAM (TriKinetics Inc Waltham, USA). These monitors were then placed 364 on an orbital shaker of $13 " \times 13^{\prime \prime}$ dimension and subjected to orbital motion of 120-rpm (unless 365 specified otherwise). Activity monitors were fixed on the orbital shaker using double-sided adhesive 366 tape, attached at the bottom of the monitor. Activity was recorded in 1-min bin and a minimum 5-min 367 of continuous quiescence was considered as sleep (Hendricks et al., 2000; Shaw et al., 2000), 368 estimated using a sliding time window. In most cases, data collected over a period of 3-days were 369 averaged and analyzed using $t$ - test or analyses of variance (ANOVA) followed by post-hoc multiple 370 comparisons using Tukey's test. To plot sleep and activity profiles, data pooled in 30-min bins and 371 averaged across 3-4-days were used. For most of the experiments flies were given OMD during 372 daytime and were left undisturbed on the shaker during night except for the experiment described in 373 Figure 1, here OMD flies were removed from the shaker at ZT12 and were placed along with 374 controls, whereas OMN flies were placed on the shaker at ZT12. At ZT00, OMD flies were placed on 375 the shaker, while OMN flies were removed and placed along with controls. OMC flies remained on 376 the shaker for the duration of $24 \mathrm{hrs}$. For perturbation experiments, at ZT04 or ZT06, experimental flies and control flies were 378 subjected to perturbation by physically shaking the monitors. For light pulse exposure under DD, 379 light of 500-lux was switched on for 10-sec without causing any disturbance to flies. To analyze the 380 effect of caffeine on sleep we used two doses of caffeine (1-mg/ml and $4-\mathrm{mg} / \mathrm{ml})$. The $1-\mathrm{mg} / \mathrm{ml}$ dose 381 did not cause fly deaths and therefore we continued with this dose for the rest of the experiments. 382 Sleep change ( $\Delta$ sleep) was estimated by subtracting mean daytime or nighttime sleep of controls 383 from daytime or nighttime sleep of each OMD fly. For figure 7d,e sleep change was calculated as 384 change in sleep of nanGAL4>dTRPAl with respect each parental control. For socio-sexual 385 interaction experiment, activity of flies was recorded in 7 -mm tubes at $28{ }^{\circ} \mathrm{C}$ instead of 5 -mm tubes 386 used for all other experiments. For the temporal silencing and activation experiments by UASshibire ${ }^{\text {ts }}$ 387 and UASATRPA1, a temperature of $28{ }^{\circ} \mathrm{C}$ was used, whereas $21^{\circ} \mathrm{C}$ was used as the baseline 388 temperature.

\section{Figure Legends:}


390 Figure 1. (a-left) Schematic representing experimental protocol used to subject flies to orbital motion.

391 DAM monitors were fixed by double sided adhesive tape on an orbital shaker that rotated the platform 392 in the horizontal plane as shown (120 rpm, unless specified). (a-right) Horizontal white and black 393 bars indicate day and night period under 12:12 h light:dark cycles. Dashed bars indicate the duration 394 for which orbital motion was given - OMD- Orbital Motion during 12h of Daytime; OMN-Orbital 395 Motion during 12h of Nighttime and OMC-Orbital Motion for the entire 24h Cycle. Sleep profile of 396 CS flies exposed to (b) OMD, (c) OMN and (d) OMC where ( $)$ indicates treated flies, and (O) 397 indicate controls. Mean sleep across 3 days averaged across flies (30-min bins) is plotted across time 398 of the day with $12 \mathrm{~h}$ light phase (white horizontal bar) and 12h dark phase (black horizontal bar). (e) 399 Mean sleep during day (unfilled bars) and night (filled bars) under OMD, OMN and OMC. Error bars 400 are SEM. ANOVA followed by post-hoc multiple comparisons Tukey's test revealed significant 401 increase $(p<0.05)$ in daytime sleep when exposed to OMD and OMC, whereas OMN has no effect $402(p>0.05)$. Asterisks denote statistical significance level of $p<0.05=*, p<0.005=* *$, and $p<0.0005=$ $403 * * *$. Nighttime sleep is not significantly altered in any regime. Sample size varied from $\mathrm{n}=24-32$ 404 flies.

405 Figure 2. (a-d) Sleep profile of (a) CS, (b) OR, (c) $w^{1118}$ and (d) $y w$ flies exposed to OMD (๑), 406 plotted along with controls (O). (e) Mean daytime sleep of controls (unfilled bars) and OMD flies 407 (filled bars). ANOVA followed by post-hoc comparisons revealed an increase in sleep $(p<0.0005)$ in $408 C S, O R, w^{1118}$, and $y w$ flies exposed to OMD. (f) Activity per waking min of OMD exposed CS, OR, $409 w^{1118}$ and $y w$ flies is comparable to controls $(p>0.05)$. (g) Daytime sleep latency is smaller for OMD 410 exposed $C S, O R$ and $y w(p<0.0005$ for $O R$ and $y w, p<0.02$ for $C S)$ but remains unchanged in $w^{1118}$ 411 flies $(p=0.16)$. (h) Nighttime sleep latency is not affected $(p>0.05)$ in any of the fly strains; All other 412 details same as Figure 1. $n=23-24$

413 Figure 3. (a) Sleep profiles of CS flies with OMD and controls, when physical disturbance was given 414 at ZT06. (b) Sleep latency post physical disturbance at ZT06 of flies from (a) showing OMD flies fall 415 asleep sooner than controls $(p<0.05)$. (c) Sleep profile of flies exposed to OMD from CT00 to CT12, 416 and received light pulse of 500-lux for 10-sec on the first day in DD at CT04. (d) Total sleep of flies 417 experiencing OMD from ZT00 to ZT04 is significantly higher $(p<0.05)$ than controls. After light 
418 pulse, sleep in OMD flies is not different from controls $(p=0.22)$. (e-left) Sleep profiles pre-treatment 419 with caffeine (pre-caf) show three sets of $C S$ flies where OMD ( $)$ and OMD-caf ( $\Delta$ ) show 420 significant increase $(p<0.05)$ in comparison to control $(\mathrm{O})$. (e-right) While OMD flies (not fed on 421 caffeine) show higher sleep levels than controls, sleep levels of flies fed with caffeine (OMD-caf) fall 422 equal to control levels. (f) Mean daytime sleep of control (white bar) OMD (black bar), OMD-caf 423 (grey bar), showing significant increase $(p<0.05)$ in sleep in response to OMD before caffeine 424 treatment (pre-caf) whereas following caffeine treatment (caf), OMD flies continue to show increase $425(p<0.0005)$ in sleep, however OMD-caf flies show similar sleep levels as controls $(p=0.98) .(\mathrm{g})$ 426 Sleep profiles of OMD and OMHD (마bital ㅆotion for Half-ㅁay between ZT00-06 only) flies plotted 427 along with controls. Mean sleep of flies in (h) showing significant increase $(p<0.0005)$ in sleep in 428 OMD and OMHD flies during the first half of the day (ZT00-ZT06), while in second half of the day 429 (ZT06-ZT12) OMHD flies sleep significantly lesser $(p<0.0005)$ than controls whereas OMD flies 430 continue to show significant increase $(p<0.0005)$.

431 Figure 4. (a-b) Sleep profiles of loss of function mutants of (a) period (per $\left.{ }^{0}\right)$ and (b) $C l o c k\left(\operatorname{Clk}^{\mathrm{Jrk}}\right)$. 432 (c) Mean daytime sleep of per $^{0}$ and (d) $c l k^{\mathrm{jrk}}$ flies with OMD is higher than controls ( $t$-test, $p<0.001$ 433 for $p e r^{0}$ and $p<0.0005$ for $c l k^{\mathrm{jrk}}$ ) whereas nighttime sleep is unaffected. (e) Activity profiles of $p e r^{0}$ 434 and (f) $c l k^{\mathrm{jrk}}$. (g) During daytime, activity counts/waking min of $p e r^{0}$ and (h) $c l k^{\mathrm{jrk}}$ flies subjected to 435 OMD did not differ from their controls $(p>0.05)$. Other details same as in Figure $1 . \mathrm{n} \geq 13$

436 Figure 5. (a-b) Sleep profiles of (a) $n a n^{d y 5}$ and (b) $n a n^{d y 5} /+$ flies. (c) Change in daytime sleep levels 437 compared to untreated controls ( $\Delta$ sleep) of $n a n^{d y 5}$ null mutants flies is similar ( $p=0.70-t$ - test) to 438 heterozygote $n a n^{d y 5} /+$ controls. (d-f) Sleep profiles of (d) $n a n G A L 4 /+$, (e) $U A S /+$ controls, and (f) 439 nan ablated nanGAL4/UAShid flies. (g) Sleep of nan-ablated neurons (black bar) is significantly 440 reduced $(p<0.0005$ for GAL4 and $p<0.01$ for $U A S$ ) compared to parental controls (GAL4 (white) and $441 U A S$ (grey)) (h-i) Sleep profiles of (h) flies with electrically silenced nan neurons, nanGAL4/UAStnt 442 (ac=active), and (i) control nanGAL4/UAStnt flies (inac=inactive). (j) sleep is significantly lower $443(p<0.01)$ in nanGAL4/UAStnt (ac) than nanGAL4/UAStnt (inac). $\mathrm{n} \geq 16$

444 Figure 6. (a-left) Sleep profiles of nompc2 flies. (a-right) Daytime sleep is significantly increased 445 ( $p<0.0001$ - $\mathrm{t}$ - test) in nompc2 flies in response to OMD compared to controls. (b-left) Sleep profiles of 
446 CS flies with antennae intact or (b-middle) surgically removed. (b-right). Sleep of intact flies and

447 flies without antennae is increased in response to the OMD $(p<0.005$ for controls and $p<0.05$ for $C S$ 448 ant ). (c-left) Sleep profiles of Orco, and (c-middle) Or83bGAL4>UASkir2.1 flies. (c-right) Daytime 449 sleep of Orco and Or83bGAL4>UASkir2.1 flies show statistically significant increase $(p<0.0001-t$ 450 test) in response to OMD compared to controls. (d-left) Sleep profile of CS flies in DD (CS-DD) and 451 (d-middle) norpA flies subjected to OMD. (d-right) Daytime sleep of CS flies is significantly greater 452 in response to OMD compared to controls $(p<0.05$ - t-test). Similarly, norpA flies show increase in 453 sleep $(p<0.0001$ - $t$ - test $)$ when exposed to OMD as compared to controls. $\mathrm{n} \geq 16$

454 Figure 7. (a-b) Sleep profiles of control (a) nanGAL4/+ and (b) nanGAL4/UASshibire $e^{\text {ts }}$ flies at $21{ }^{\circ} \mathrm{C}$ 455 and $28{ }^{\circ} \mathrm{C}$. (c) Daytime sleep shows similar increase ( $p=0.95$, ANOVA followed by Tukey's test) in 456 response to OMD in both the genotypes at $21{ }^{\circ} \mathrm{C}$. At $28{ }^{\circ} \mathrm{C}$ silenced show statistically significant 457 difference $(p<0.0005)$ in change in daytime sleep. At $28{ }^{\circ} \mathrm{C}$, nighttime sleep is also reduced $(p<0.05)$ 458 in silenced flies compared to controls. (d) Sleep profiles of control (nanGAL4/+) and nan activated 459 (nanGAL4/UASdTRPAl) flies. (e) Daytime sleep in two genotypes does not differ at $21^{\circ} \mathrm{C}$, however, 460 when temperature is increased to $28{ }^{\circ} \mathrm{C}$, nanGAL4/UASdTRPAl flies show increase in sleep $(p<0.05$ 461 for nanGAL4/+ and and $p<0.005$ for $d T R P A 1 /+$-ANOVA followed by Tukey's test). (f) Sleep 462 profiles of of nanGALA/UASATRPA1 males cohoused with CS females, across two days. Temperature 463 was $28{ }^{\circ} \mathrm{C}$ for the first two days and then isolated males (without partners) were returned to $21{ }^{\circ} \mathrm{C}$ for 464 two days. (g) average sleep profile at $28^{\circ} \mathrm{C}(\mathrm{h})$ Sleep bar graph of nanGAL4/UASdTRPA1 in different 465 combination (M, MM, and MF, with F being CS instead of nanGAL4/UASdTRPA1). Daytime sleep of 466 nanGAL4/UASdTRPA1 show decrease in sleep compared to controls kept solitary (M) or in group of 467 two (MM). (h) Day as well nighttime sleep in nanGAL4/UASdTRPAl male and CS female is 468 significantly decreased $(p<0.0001)$ compared to those in nanGAL4/UASdTRPA1 males maintained in 469 pairs or solitarily.. All other details same as in Figure 1. $\mathrm{n} \geq 16$

470 Figure 8. (a-b) Sleep profiles of (a) $c 929 / d O R K \triangle-N C$ and (b) $c 929 / N a C h B a c$ flies. (c) Daytime 471 sleep of $c 929 / \mathrm{NaChBac}$ is lower ( $p<0.005$ for $c 929 / d O R K \triangle-N C$ and $p<0.05$ for $c 929 G A L 4$ ) than $472 c 929 / d O R K \triangle-N C$ and $c 929 G A L 4$. (d-e) Sleep profiles of (d) $c 929 G A L 4 /+$ and (e) 473 c929GAL4/UASdTRPA1 flies. (f) Daytime sleep of all three genotypes viz. c929GAL4/UASdTRPA1, 
474

475

476

477

478

479

480

481

482

483

484

485

486

487

488

489

490

491

492

493

494

495

496

497

498

499

500

501

502

503

504

505

506

507

508

509

$c 929 G A L 4 /+$ and UASdTRPA1/+ show similar $(p>0.91)$ at $21^{\circ} \mathrm{C}$ in response to OMD, whereas at 28

${ }^{\circ} \mathrm{C}, c 929 G A L 4 / U A S d T R P A 1$ flies shows statistically significant decrease in change in sleep $(p<0.001$, $p<0.05$ in comparison to $c 929 G A L 4 /+$ and $U A S d T R P A 1 /+$ respectively-ANOVA followed by Tukey's tests) in comparison to the other two genotypes. All other details same as in Figure 1. $n \geq 31$

\section{References:}

1. Allada, R., \& Siegel, J. M. (2008). Unearthing the phylogenetic roots of sleep. Current biology, 18(15), R670-R679.

2. Andretic R, Shaw PJ. (2005). Essentials of sleep recordings in Drosophila: moving beyond sleep time. Methods in Enzymology. 393:759-72.

3. Bayer, L., Constantinescu, I., Perrig, S., Vienne, J., Vidal, P. P., Mühlethaler, M., \& Schwartz, S. (2011). Rocking synchronizes brain waves during a short nap. Current Biology, 21(12), 461-R462.

4. Bellesi, M., Riedner, B. A., Garcia-Molina, G. N., Cirelli, C., \& Tononi, G. (2014). Enhancement of sleep slow waves: underlying mechanisms and practical consequences. Frontiers in systems neuroscience, 8, 208.

5. Bloomquist, B. T., Shortridge, R. D., Schneuwly, S., Perdew, M., Montell, C., Steller, H., Rubin G \& Pak, W. L. (1988). Isolation of a putative phospholipase C gene of Drosophila, norpA, and its role in phototransduction. Cell, 54(5), 723-733.

6. Borbély AA (1982). A two process model of sleep regulation. Human Neurobiology. 1(3): 195-204.

7. Borbély AA, Daan S, Wirz-Justice A, Deboer T. (2016). The two-process model of sleep regulation: a reappraisal. Journal of Sleep Research. 25(2): 131-43.

8. Campbell, S. S., \& Tobler, I. (1984). Animal sleep: a review of sleep duration across phylogeny. Neuroscience \& Biobehavioral Reviews, 8(3), 269-300.

9. Crocker, A., \& Sehgal, A. (2010). Genetic analysis of sleep. Genes \& development, 24(12), 1220-1235.

10. Donelson, N. C., \& Sanyal, S. (2015). Use of Drosophila in the investigation of sleep disorders. Experimental neurology, 274, 72-79.

11. Donlea, J. M., Ramanan, N., \& Shaw, P. J. (2009). Use-dependent plasticity in clock neurons regulates sleep need in Drosophila. Science, 324(5923), 105-108.

12. Donlea, J. M., Thimgan, M. S., Suzuki, Y., Gottschalk, L., \& Shaw, P. J. (2011). Inducing sleep by remote control facilitates memory consolidation in Drosophila. Science, 332(6037), 1571-1576.

13. Effertz, T., Wiek, R., \& Göpfert, M. C. (2011). NompC TRP channel is essential for Drosophila sound receptor function. Current Biology, 21(7), 592-597. 
510

511

512

513

514

515

516

517

518

519

520

521

522

523

524

525

526

527

528

529

530

531

532

533

534

535

536

537

538

539

540

541

542

543

544

545
14. Fontanini, A., \& Bower, J. M. (2006). Slow-waves in the olfactory system: an olfactory perspective on cortical rhythms. Trends in neurosciences, 29(8), 429-437.

15. Fujii, S., Krishnan, P., Hardin, P., \& Amrein, H. (2007). Nocturnal male sex drive in Drosophila. Current Biology, 17(3), 244-251.

16. Ganguly-Fitzgerald, I., Donlea, J., \& Shaw, P. J. (2006). Waking experience affects sleep need in Drosophila. Science, 313(5794), 1775-1781.

17. Gong, Z., Son, W., Chung, Y. D., Kim, J., Shin, D. W., McClung, C. A., Lee Y., Lee HW., Chang DJ., Kaang BK., Oh U., Hirsh J., Kernan MJ., Kim C., \& Cho, H. (2004). Two interdependent TRPV channel subunits, inactive and Nanchung, mediate hearing in Drosophila. Journal of Neuroscience, 24(41), 9059-9066.

18. Goodwin, S. F., Taylor, B. J., Villella, A., Foss, M., Ryner, L. C., Baker, B. S., \& Hall, J. C. (2000). Aberrant splicing and altered spatial expression patterns in fruitless mutants of Drosophila melanogaster. Genetics, 154(2), 725-745.

19. Guo, F., Yu, J., Jung, H. J., Abruzzi, K. C., Luo, W., Griffith, L. C., \& Rosbash, M. (2016). Circadian neuron feedback controls the Drosophila sleep-activity profile. Nature, 536(7616), 292.

20. Hamada, F. N., Rosenzweig, M., Kang, K., Pulver, S. R., Ghezzi, A., Jegla, T. J., \& Garrity, P. A. (2008). An internal thermal sensor controlling temperature preference in Drosophila. Nature, 454(7201), 217.

21. Hendricks, J. C., Finn, S. M., Panckeri, K. A., Chavkin, J., Williams, J. A., Sehgal, A., \& Pack, A. I. (2000). Rest in Drosophila is a sleep-like state. Neuron, 25(1), 129-138.

22. Huber R, Hill SL, Holladay C, Biesiadecki M, Tononi G, Cirelli C. (2004). Sleep homeostasis in Drosophila melanogaster. Sleep. 27(4): 628-39.

23. Kamikouchi, A., Inagaki, H. K., Effertz, T., Hendrich, O., Fiala, A., Göpfert, M. C., \& Ito, K. (2009). The neural basis of Drosophila gravity-sensing and hearing. Nature, 458(7235), 165.

24. Keene, A. C., Duboué, E. R., McDonald, D. M., Dus, M., Suh, G. S., Waddell, S., \& Blau, J. (2010). Clock and cycle limit starvation-induced sleep loss in Drosophila. Current Biology, 20(13), 1209-1215.

25. Kim, J., Chung, Y. D., Park, D. Y., Choi, S., Shin, D. W., Soh, H., Lee HW, Son W, Yim J, Park CS., Kim C.,\& Kernan, M. J. (2003). A TRPV family ion channel required for hearing in Drosophila. Nature, 424(6944), 81.

26. Kompotis, K., Hubbard, J., Emmenegger, Y., Perrault, A., Mühlethaler, M., Schwartz, S., Bayer L., \& Franken, P. (2019). Rocking promotes sleep in mice through rhythmic stimulation of the vestibular system. Current Biology, 29(3), 392-401.

27. Kung, C. (2005). A possible unifying principle for mechanosensation. Nature, 436(7051), 647. 
546

547

548

549

550

551

552

553

554

555

556

557

558

559

560

561

562

563

564

565

566

567

568

569

570

571

572

573

574

575

576

577

578

579

580

581

28. Kunst, M., Hughes, M. E., Raccuglia, D., Felix, M., Li, M., Barnett, G., Duah J., \& Nitabach, M. N. (2014). Calcitonin gene-related peptide neurons mediate sleep-specific circadian output in Drosophila. Current Biology, 24(22), 2652-2664.

29. Lamaze A, Krätschmer P, Chen KF, Lowe S, Jepson JEC. (2018). A Wake-Promoting Circadian Output Circuit in Drosophila. Current Biology. 28(19):3098-3105.

30. Lamaze, A., Öztürk-Çolak, A., Fischer, R., Peschel, N., Koh, K., \& Jepson, J. E. (2017). Regulation of sleep plasticity by a thermo-sensitive circuit in Drosophila. Scientific reports, 7 , 40304.

31. Larsson MC, Domingos AI, Jones WD, Chiappe ME, Amrein H, Vosshall LB. (2004). Or83b encodes a broadly expressed odorant receptor essential for Drosophila olfaction. Neuron. 43(5):703-14.

32. Linford, N. J., Chan, T. P., \& Pletcher, S. D. (2012). Re-patterning sleep architecture in Drosophila through gustatory perception and nutritional quality. PLoS genetics, 8(5), e1002668.

33. Liu, S., Liu, Q., Tabuchi, M., \& Wu, M. N. (2016). Sleep drive is encoded by neural plastic changes in a dedicated circuit. Cell, 165(6), 1347-1360.

34. Lone, S. R., \& Sharma, V. K. (2012). Or47b receptor neurons mediate sociosexual interactions in the fruit fly Drosophila melanogaster. Journal of biological rhythms, 27(2), 107-116.

35. Lone, S. R., Potdar, S., Srivastava, M., \& Sharma, V. K. (2016). Social experience is sufficient to modulate sleep need of Drosophila without increasing wakefulness. PloS one, 11(3), e0150596.

36. Marshall, L., Helgadóttir, H., Mölle, M., \& Born, J. (2006). Boosting slow oscillations during sleep potentiates memory. Nature, 444(7119), 610.

37. Massimini, M., Ferrarelli, F., Esser, S. K., Riedner, B. A., Huber, R., Murphy, M., Perterson M.J., \& Tononi, G. (2007). Triggering sleep slow waves by transcranial magnetic stimulation. Proceedings of the National Academy of Sciences, 104(20), 8496-8501.

38. Morita, E., Imai, M., Okawa, M., Miyaura, T., \& Miyazaki, S. (2011). A before and after comparison of the effects of forest walking on the sleep of a community-based sample of people with sleep complaints. BioPsychoSocial medicine, 5(1), 13.

39. Parisky, K. M., Agosto, J., Pulver, S. R., Shang, Y., Kuklin, E., Hodge, J. J., White B.H., \& Griffith, L. C. (2008). PDF cells are a GABA-responsive wake-promoting component of the Drosophila sleep circuit. Neuron, 60(4), 672-682.

40. Parisky, K. M., Rivera, J. L. A., Donelson, N. C., Kotecha, S., \& Griffith, L. C. (2016). Reorganization of sleep by temperature in Drosophila requires light, the homeostat, and the circadian clock. Current Biology, 26(7), 882-892. 
582

583

584

585

586

587

588

589

590

591

592

593

594

595

596

597

598

599

600

601

602

603

604

605

606

607

608

609

610

611

612

613

614

615

616

617

41. Perrault, A. A., Khani, A., Quairiaux, C., Kompotis, K., Franken, P., Muhlethaler, M., Schwartz S., \& Bayer, L. (2019). Whole-night continuous rocking entrains spontaneous neural oscillations with benefits for sleep and memory. Current Biology, 29(3), 402-411.

42. Pompeiano, O., \& Swett, J. E. (1962). EEG and behavioral manifestations of sleep induced by cutaneous nerve stimulation in normal cats. Archives italiennes de biologie.

43. Potdar, S., \& Sheeba, V. (2013). Lessons from sleeping flies: insights from Drosophila melanogaster on the neuronal circuitry and importance of sleep. Journal of neurogenetics, 27(1-2), 23-42.

44. Ramdya, P., Lichocki, P., Cruchet, S., Frisch, L., Tse, W., Floreano, D., \& Benton, R. (2015). Mechanosensory interactions drive collective behaviour in Drosophila. Nature, 519(7542), 233.

45. Reato, D., Gasca, F., Datta, A., Bikson, M., Marshall, L., \& Parra, L. C. (2013). Transcranial electrical stimulation accelerates human sleep homeostasis. PLoS computational biology, 9(2), e1002898.

46. Saper, C. B., Fuller, P. M., Pedersen, N. P., Lu, J., \& Scammell, T. E. (2010). Sleep state switching. Neuron, 68(6), 1023-1042.

47. Sehadova, H., Glaser, F. T., Gentile, C., Simoni, A., Giesecke, A., Albert, J. T., \& Stanewsky, R. (2009). Temperature entrainment of Drosophila's circadian clock involves the gene nocte and signaling from peripheral sensory tissues to the brain. Neuron, 64(2), 251-266.

48. Simpson JH (2009). Mapping and manipulating neural circuits in the fly brain. Advances in Genetics. 65:79-143.

49. Shang, Y., Donelson, N. C., Vecsey, C. G., Guo, F., Rosbash, M., \& Griffith, L. C. (2013). Short neuropeptide F is a sleep-promoting inhibitory modulator. Neuron, 80(1), 171-183.

50. Shang, Y., Griffith, L. C., \& Rosbash, M. (2008). Light-arousal and circadian photoreception circuits intersect at the large PDF cells of the Drosophila brain. Proceedings of the National Academy of Sciences, 105(50), 19587-19594.

51. Shang, Y., Haynes, P., Pírez, N., Harrington, K. I., Guo, F., Pollack, J., Pengyu Hong, Leslie C. Griffith., \& Rosbash, M. (2011). Imaging analysis of clock neurons reveals light buffers the wake-promoting effect of dopamine. Nature neuroscience, 14(7), 889.

52. Shaw, P. J., Cirelli, C., Greenspan, R. J., \& Tononi, G. (2000). Correlates of sleep and waking in Drosophila melanogaster. Science, 287(5459), 1834-1837.

53. Shaw, P., Tafti, M., \& Thorpy, M. J. (Eds.). (2013). The genetic basis of sleep and sleep disorders. Cambridge University Press.

54. Sheeba, V., Fogle, K. J., Kaneko, M., Rashid, S., Chou, Y. T., Sharma, V. K., \& Holmes, T. C. (2008). Large ventral lateral neurons modulate arousal and sleep in Drosophila. Current Biology, 18(20), 1537-1545. 
618

619

620

621

622

623

624

625

626

627

628

629

630

631

632

633

634

635

636

637

638

639

640

641

642

643

644

645

646

647

648

649

55. Siebern, A. T., Suh, S., \& Nowakowski, S. (2012). Non-pharmacological treatment of insomnia. Neurotherapeutics : the journal of the American Society for Experimental NeuroTherapeutics, 9(4), 717-727.

56. Siegel, J. M. (2009). Sleep viewed as a state of adaptive inactivity. Nature Reviews Neuroscience, 10(10), 747.

57. Simoni, A., Wolfgang, W., Topping, M. P., Kavlie, R. G., Stanewsky, R., \& Albert, J. T. (2014). A mechanosensory pathway to the Drosophila circadian clock. Science, 343(6170), $525-528$.

58. Tang, H. Y. J., Riegel, B., McCurry, S. M., \& Vitiello, M. V. (2016). Open-loop audio-visual stimulation (AVS): A useful tool for management of insomnia?. Applied psychophysiology and biofeedback, 41(1), 39-46.

59. Tononi G., Riedner B. A., Hulse B. K., Ferrarelli F., Sarasso S. (2010). Enhancing sleep slow waves with natural stimuli. Medicamundi 54, 73-79

60. Tuthill, J. C., \& Wilson, R. I. (2016). Mechanosensation and adaptive motor control in insects. Current Biology, 26(20), R1022-R1038.

61. Van Alphen, B., Yap, M. H., Kirszenblat, L., Kottler, B., \& van Swinderen, B. (2013). A dynamic deep sleep stage in Drosophila. Journal of Neuroscience, 33(16), 6917-6927.

62. Venken, K. J., Simpson, J. H., \& Bellen, H. J. (2011). Genetic manipulation of genes and cells in the nervous system of the fruit fly. Neuron, 72(2), 202-230.

63. Viswanath, V., Story, G. M., Peier, A. M., Petrus, M. J., Lee, V. M., Hwang, S. W., Patapoutain A., \& Jegla, T. (2003). Ion channels: Opposite thermosensor in fruitfly and mouse. Nature, 423(6942), 822.

64. Walker, R. G., Willingham, A. T., \& Zuker, C. S. (2000). A Drosophila mechanosensory transduction channel. Science, 287(5461), 2229-2234.

65. Woodward, S., Tauber, E. S., Spielman, A. J., \& Thorpy, M. J. (1990). Effects of otolithic vestibular stimulation on sleep. Sleep, 13(6), 533-537.

66. Yadlapalli, S., Jiang, C., Bahle, A., Reddy, P., Meyhofer, E., \& Shafer, O. T. (2018). Circadian clock neurons constantly monitor environmental temperature to set sleep timing. Nature, 555(7694), 98.

67. Yan, Z., Zhang, W., He, Y., Gorczyca, D., Xiang, Y., Cheng, L. E., Meltzer S., Jen L.Y.,\& Jan, Y. N. (2013). Drosophila NOMPC is a mechanotransduction channel subunit for gentletouch sensation. Nature, 493(7431), 221. 
a
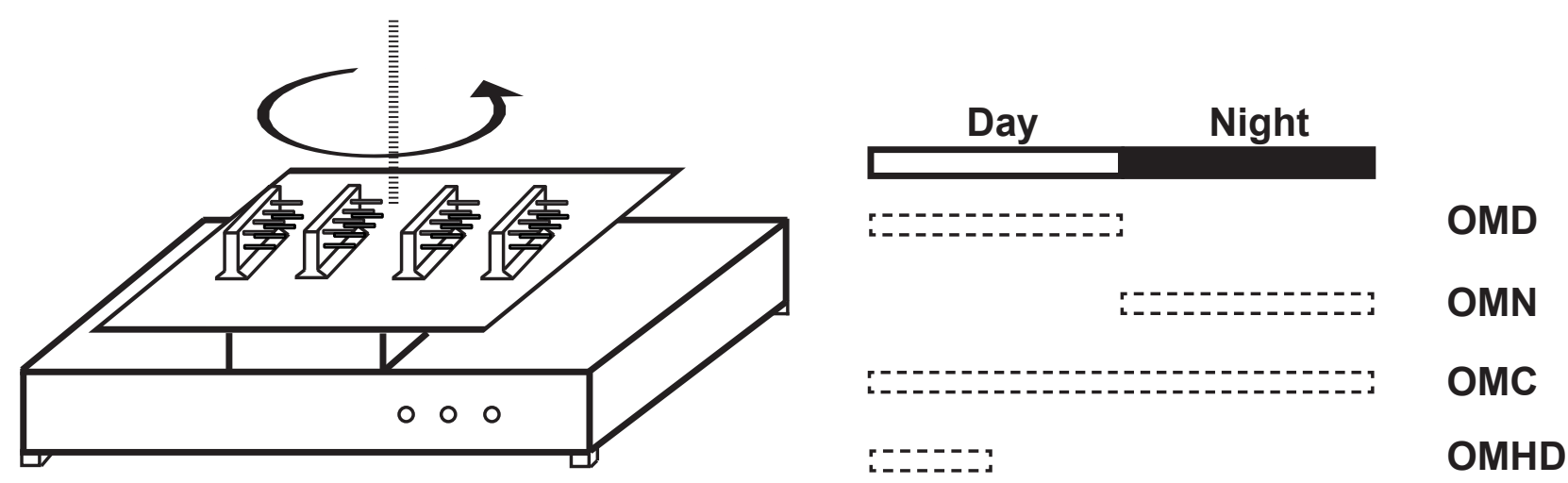

b
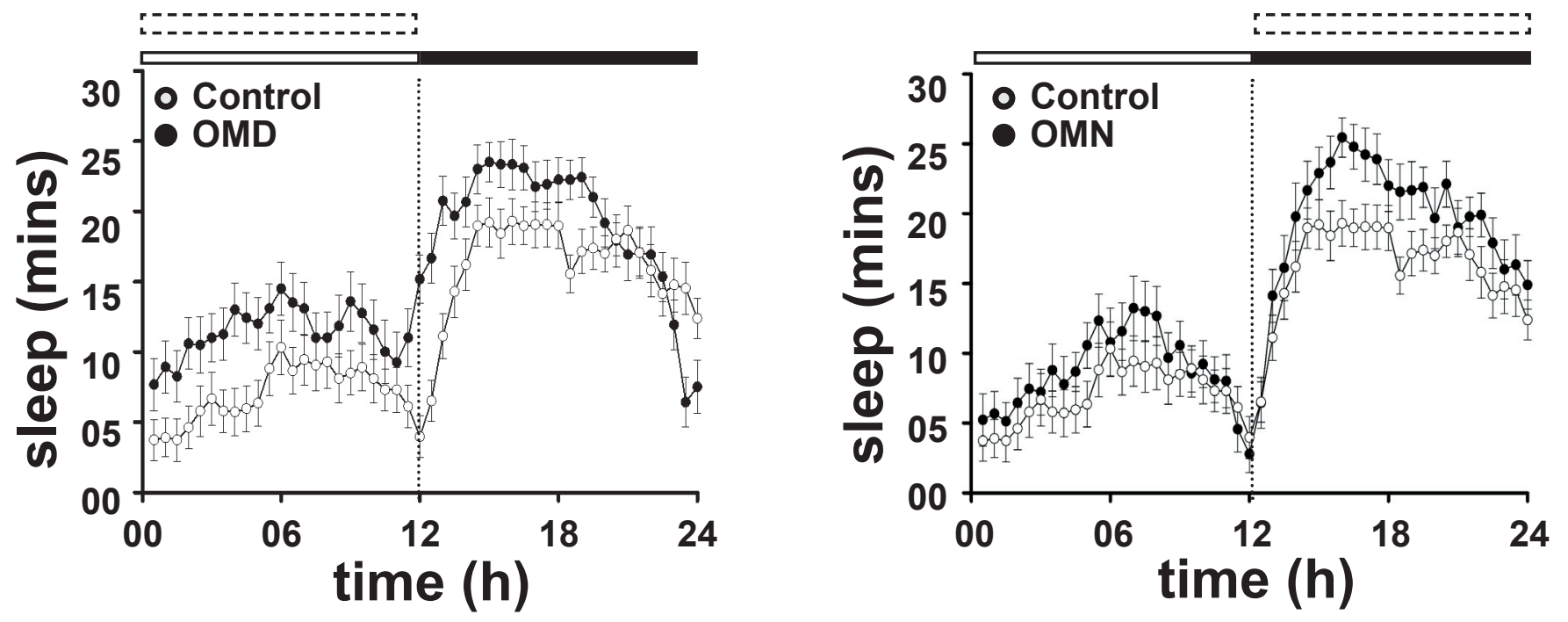

d
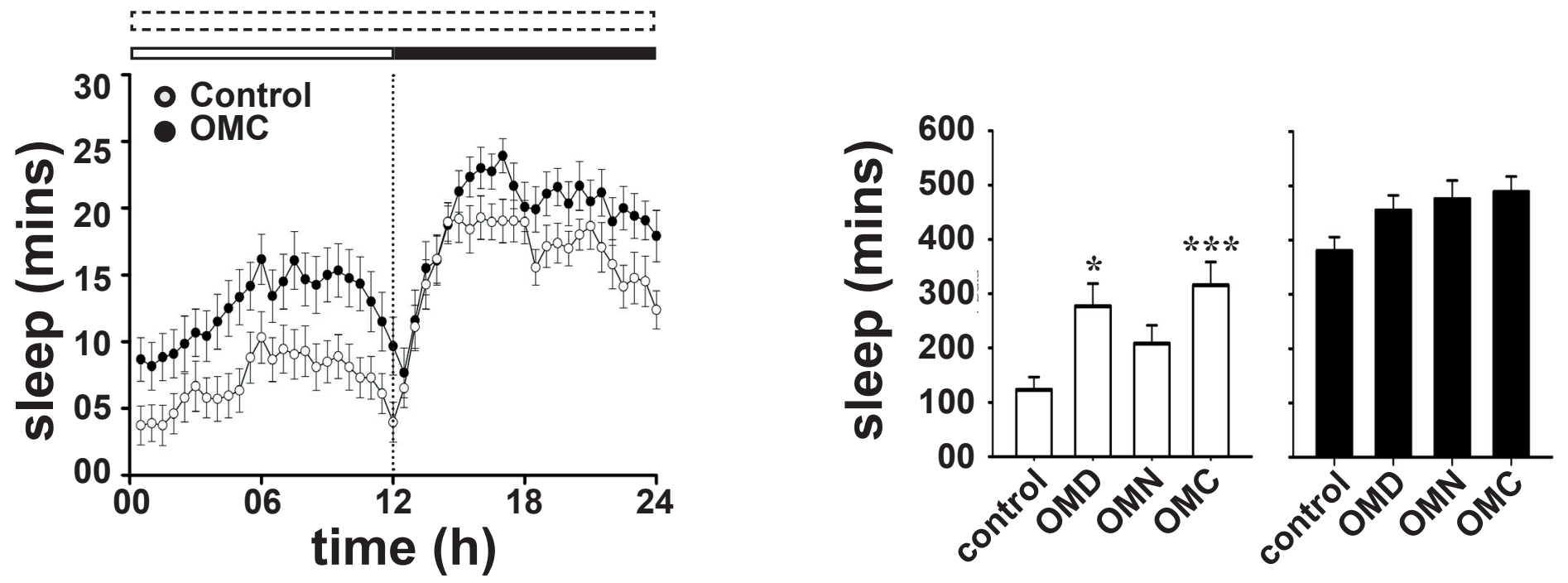
a

bioRxiv preprint doi: https://doi.org/10.1101/829861; this version posted November 5, 2019. The copyright holder for this preprint (which was not certified by peer review) is the author/funder. All rights reserved. No reuse allowed without permissio

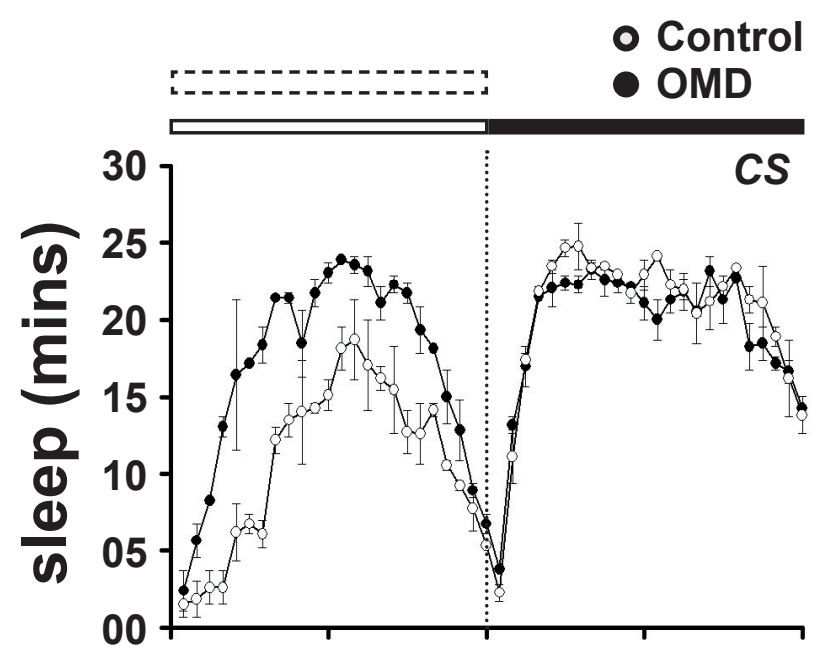

e

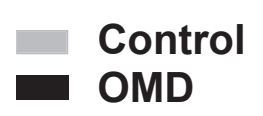

b

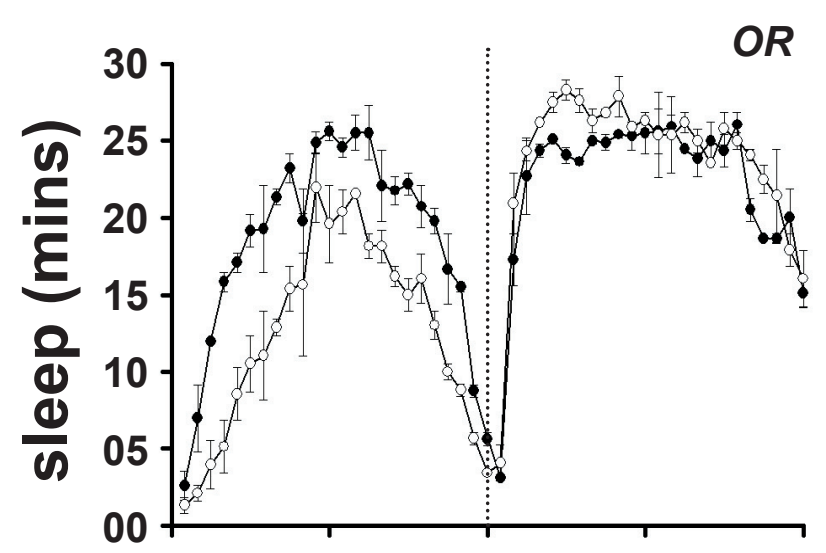

C

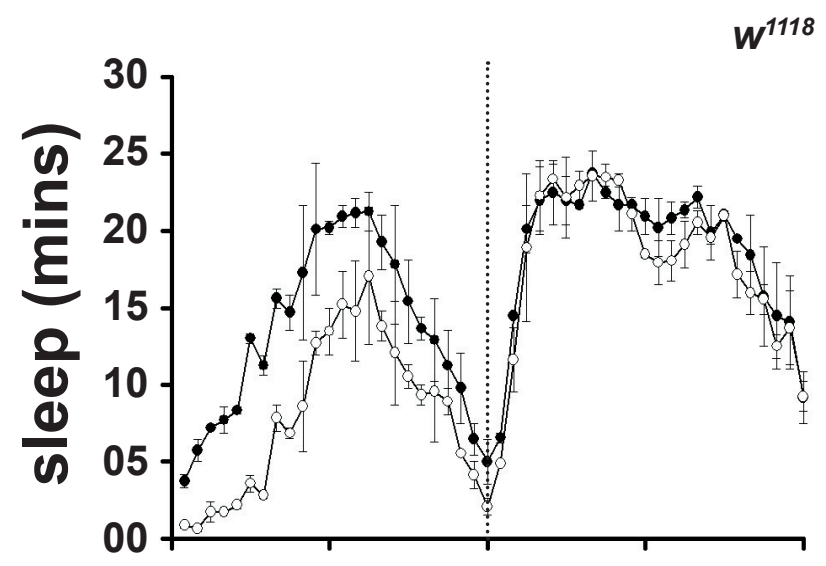

d

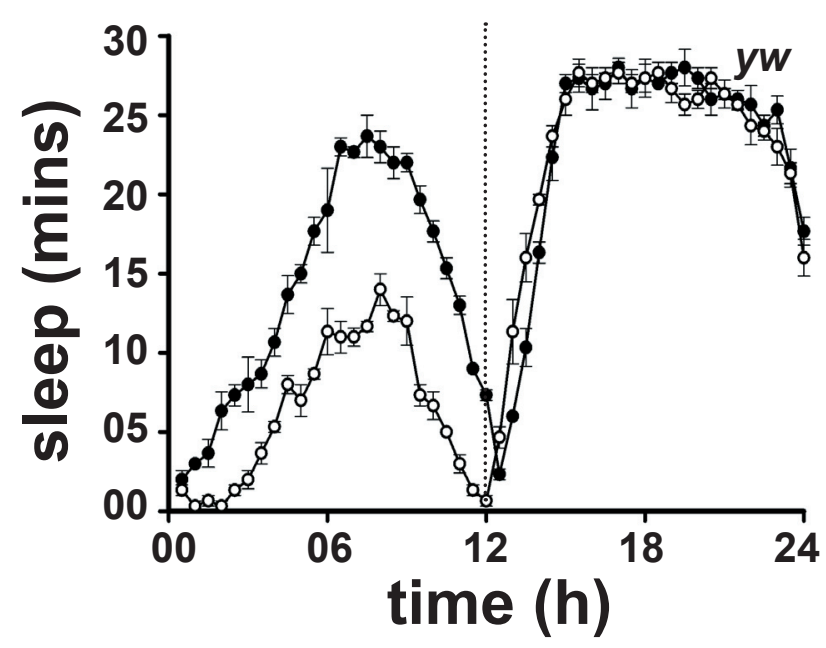

g
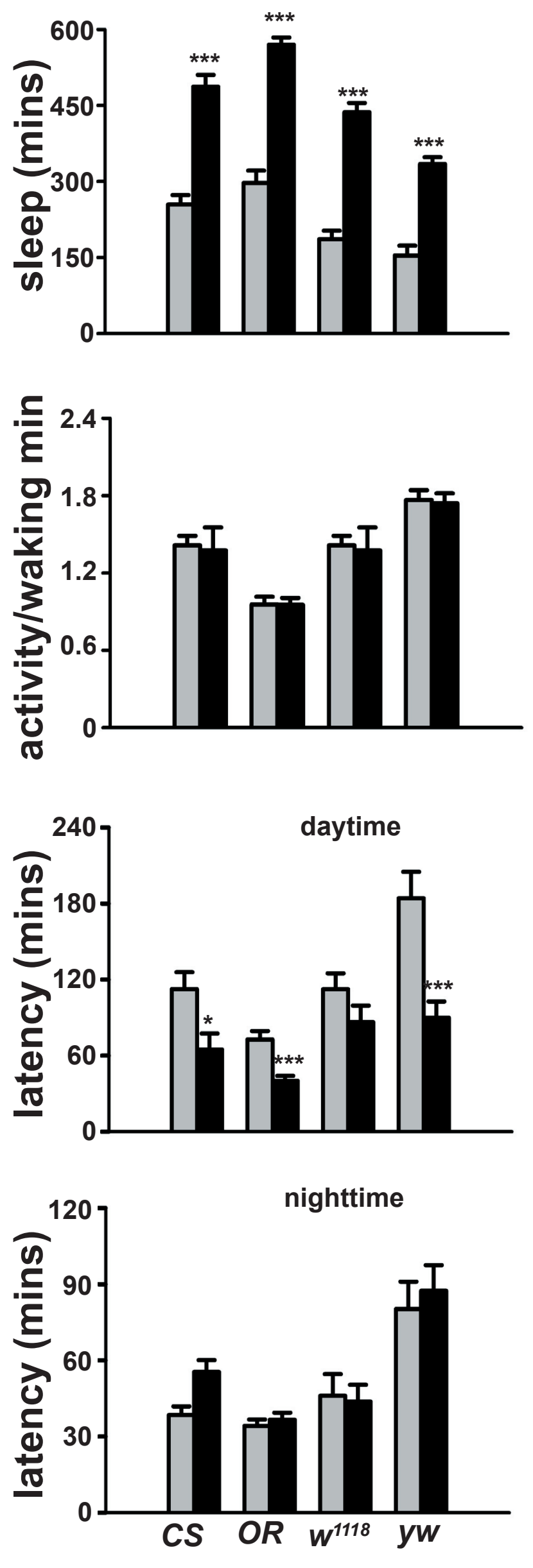
a

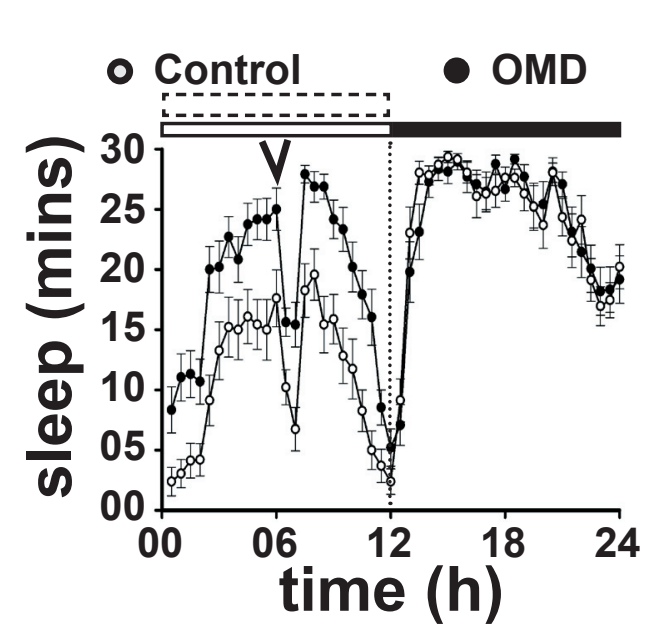

b

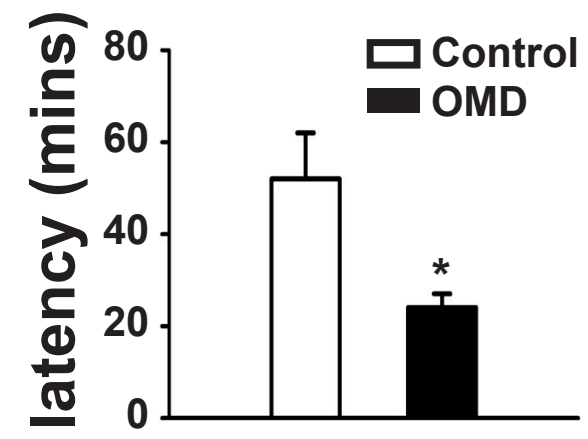

C

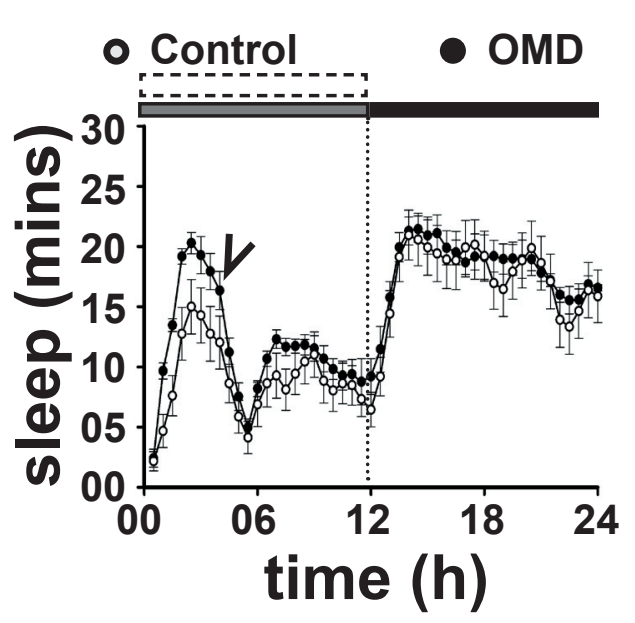

d
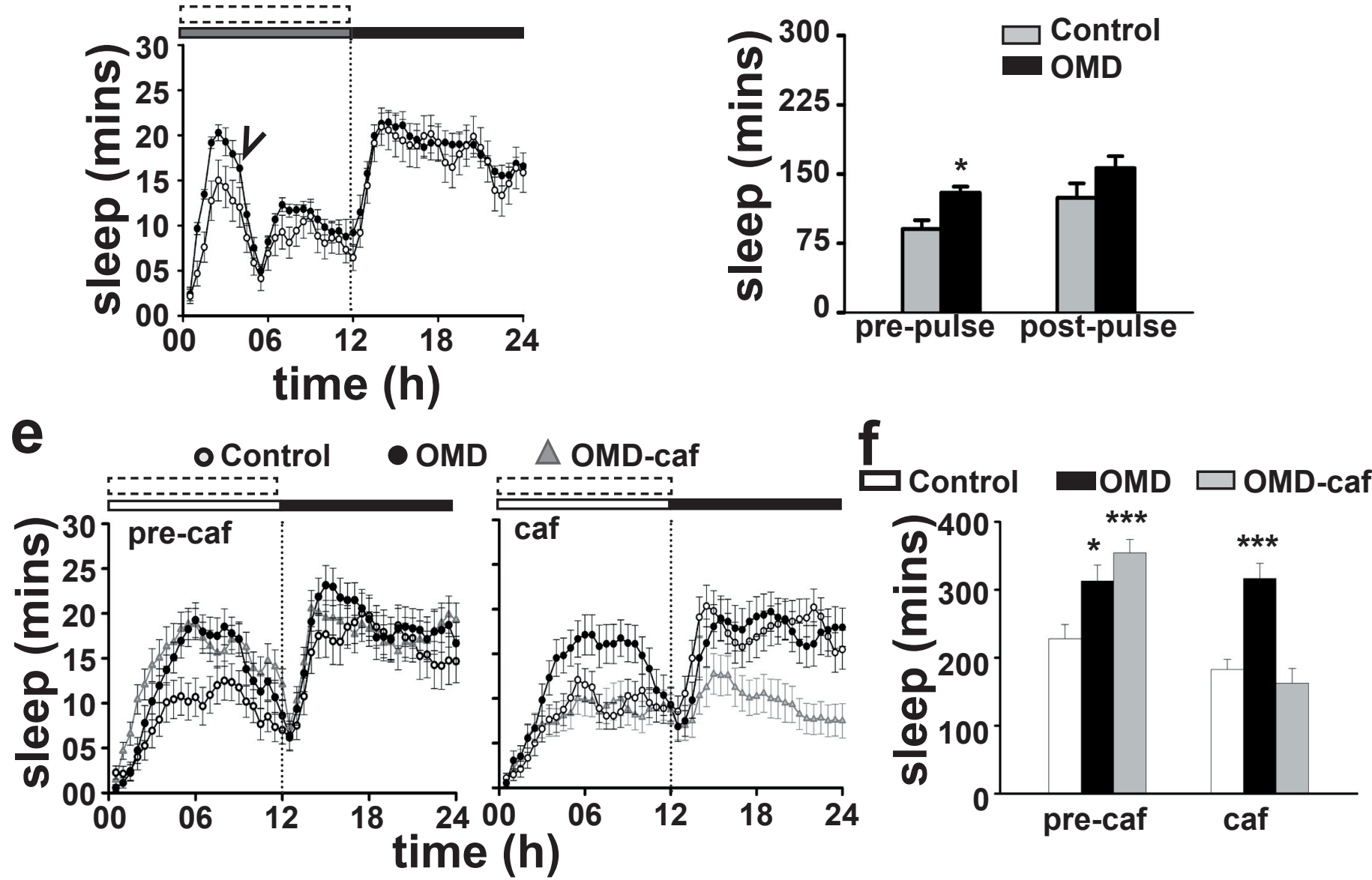

$g$
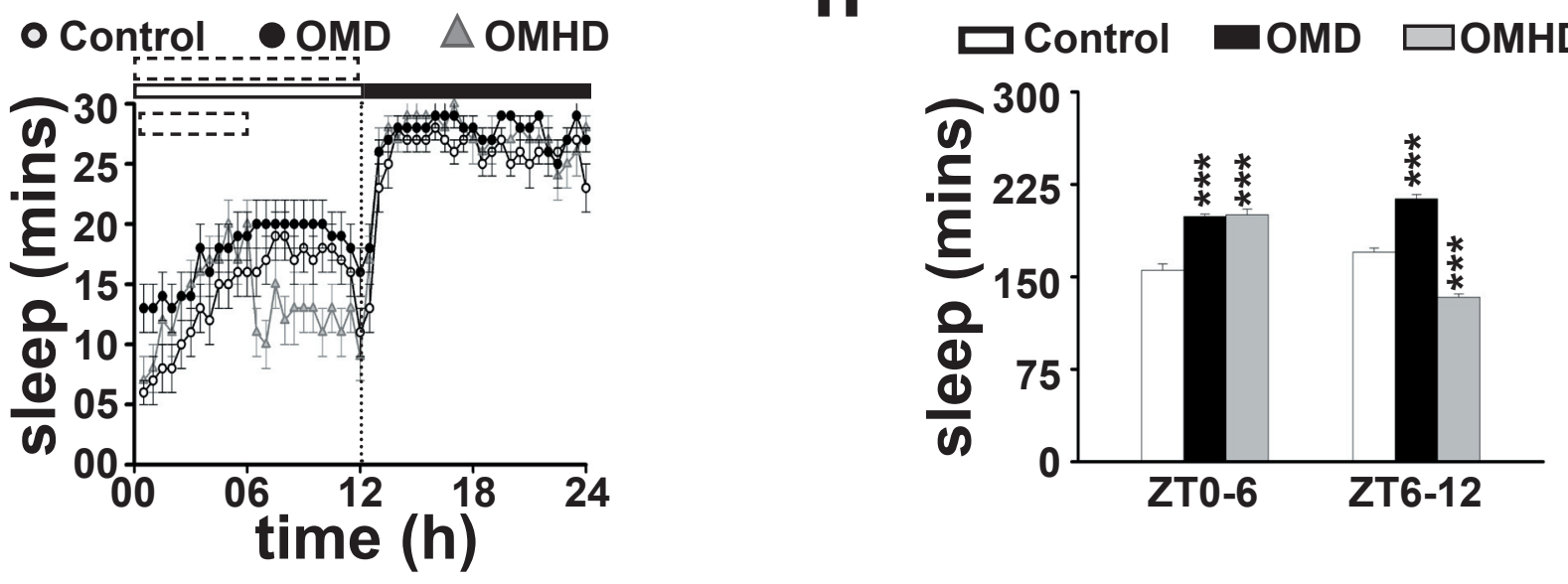
Figure 4

a

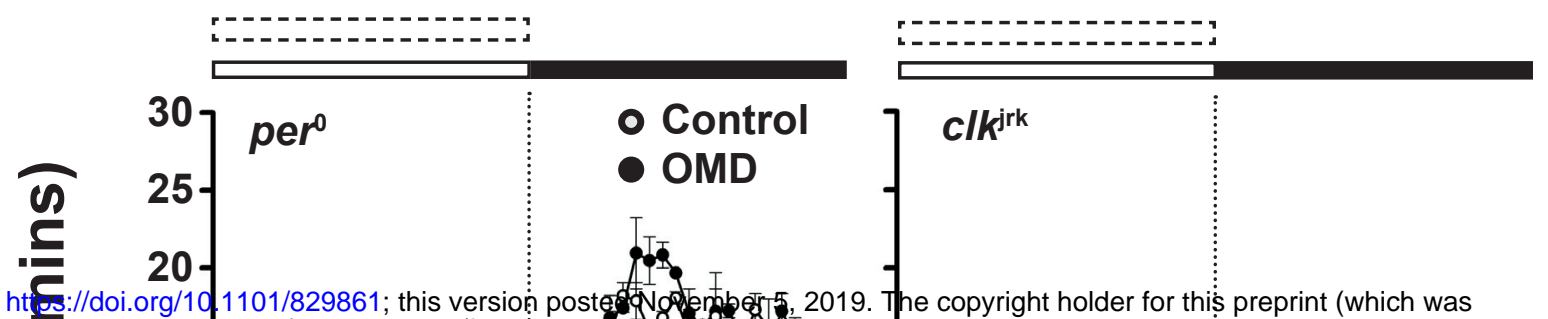

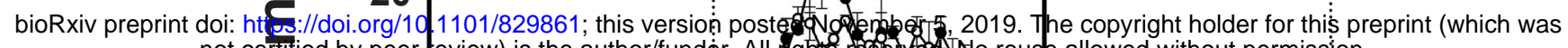

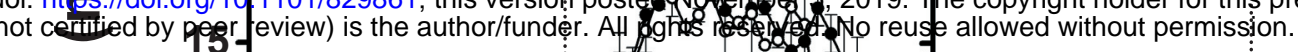

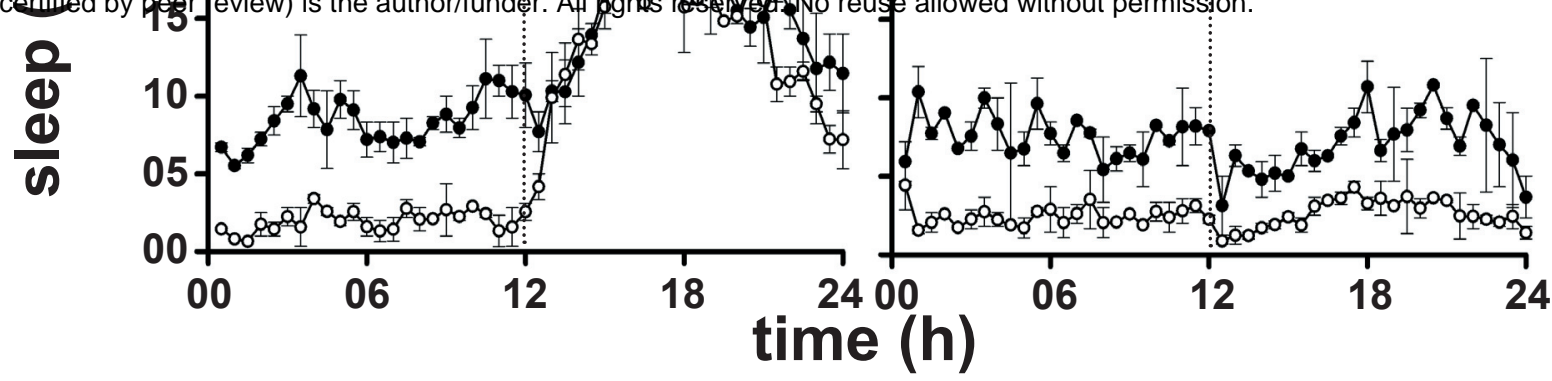

b

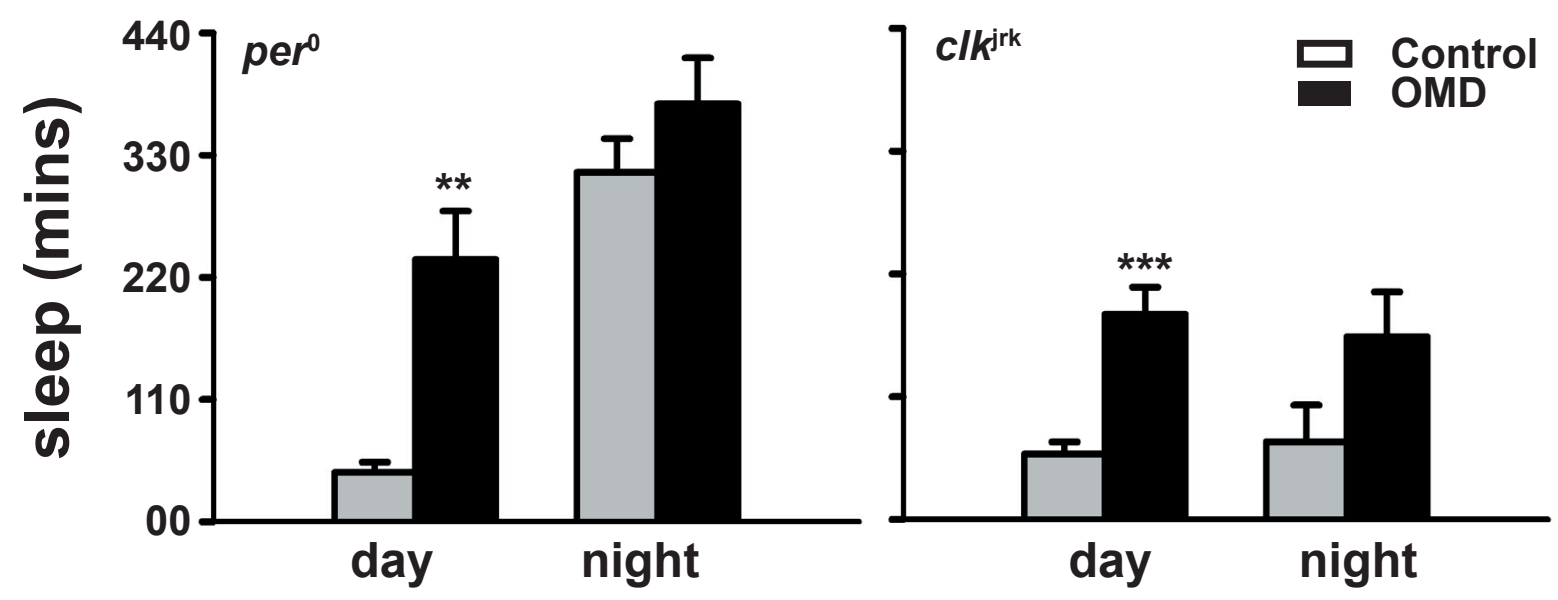

C

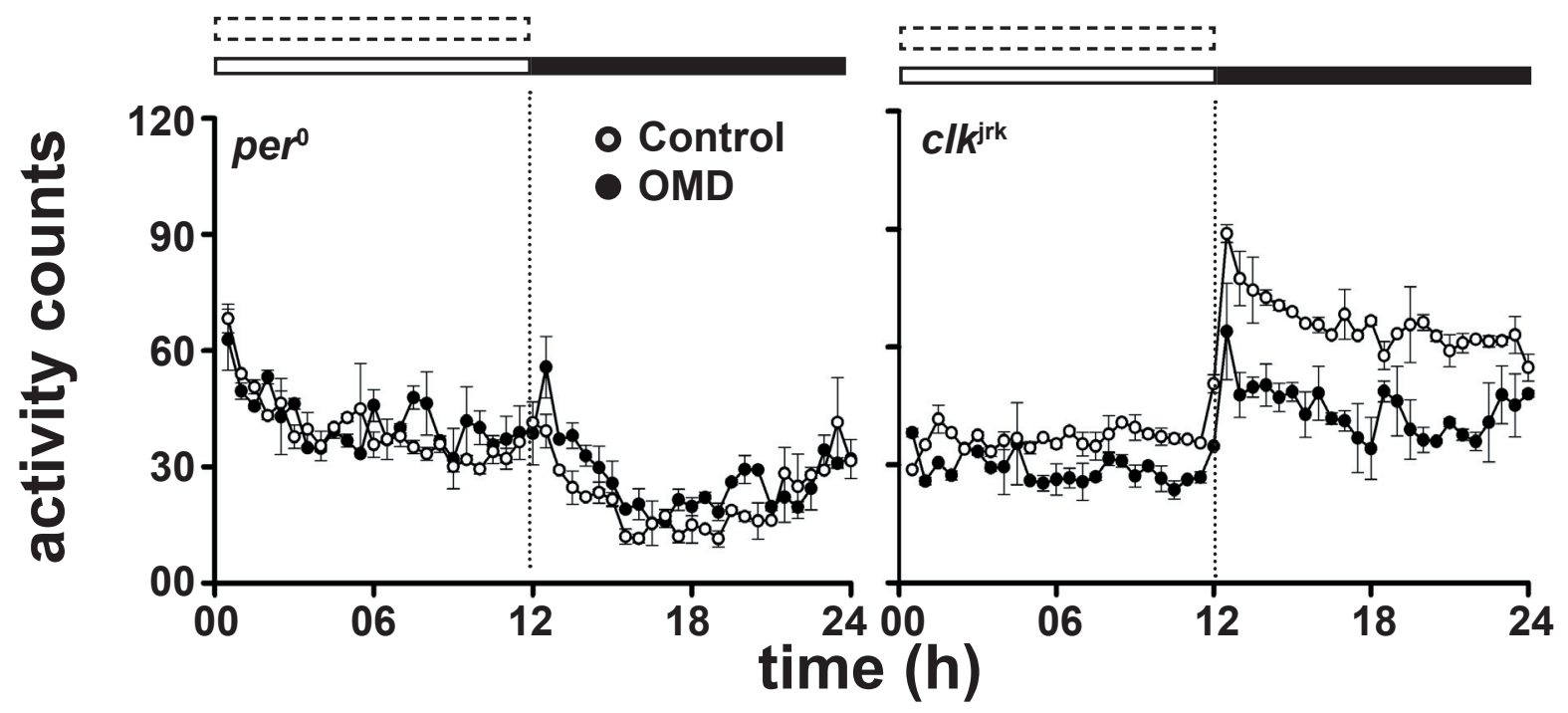

d

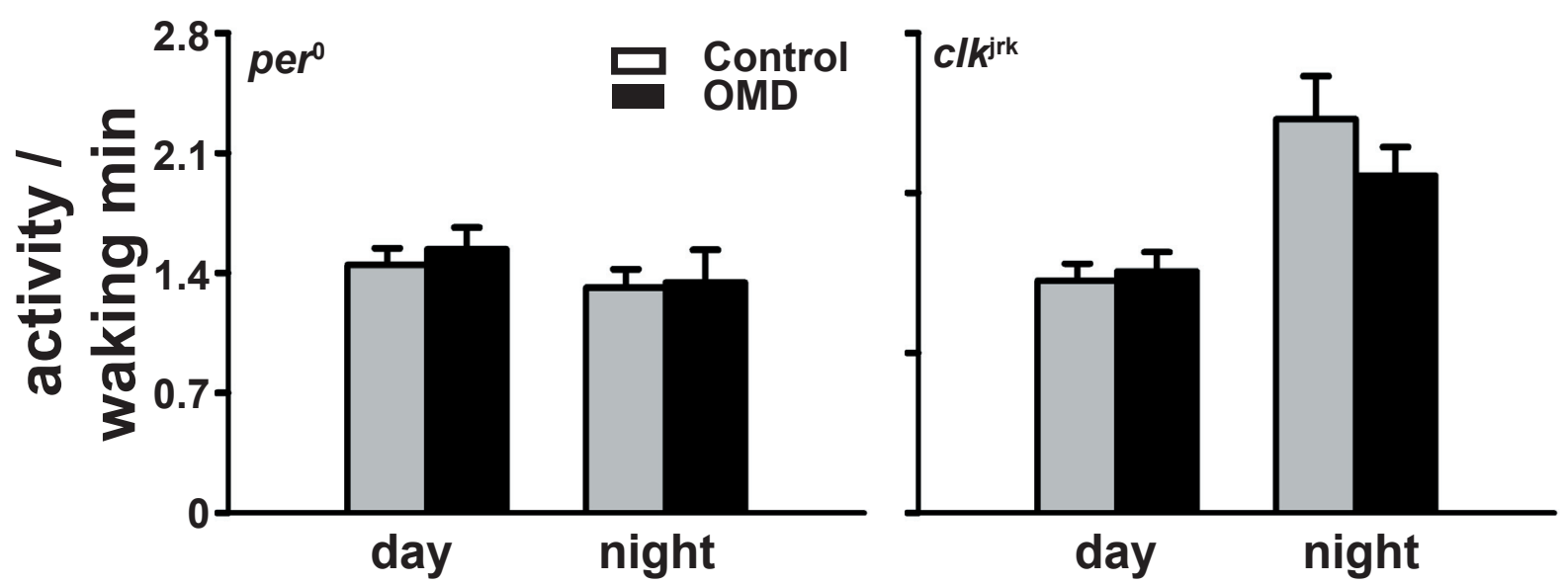


a

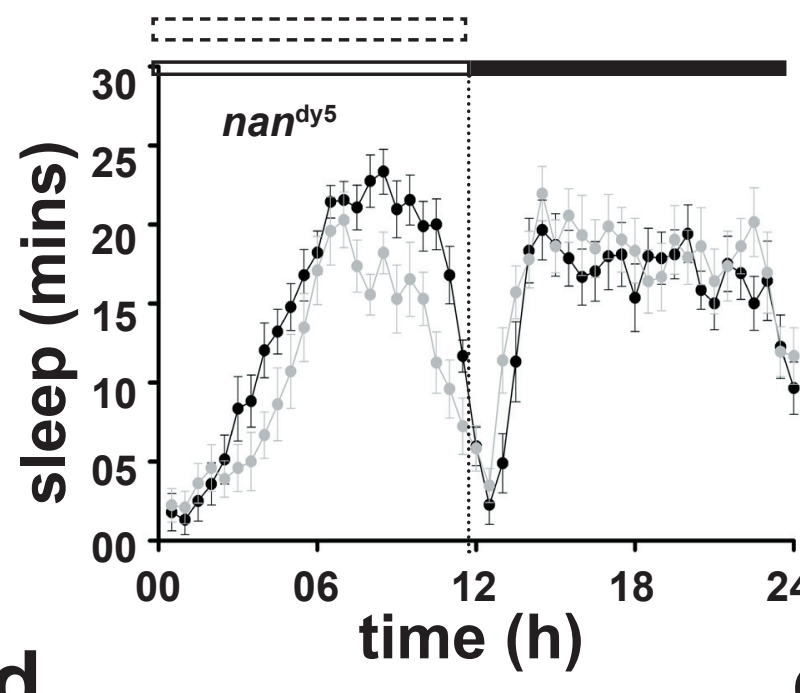

d
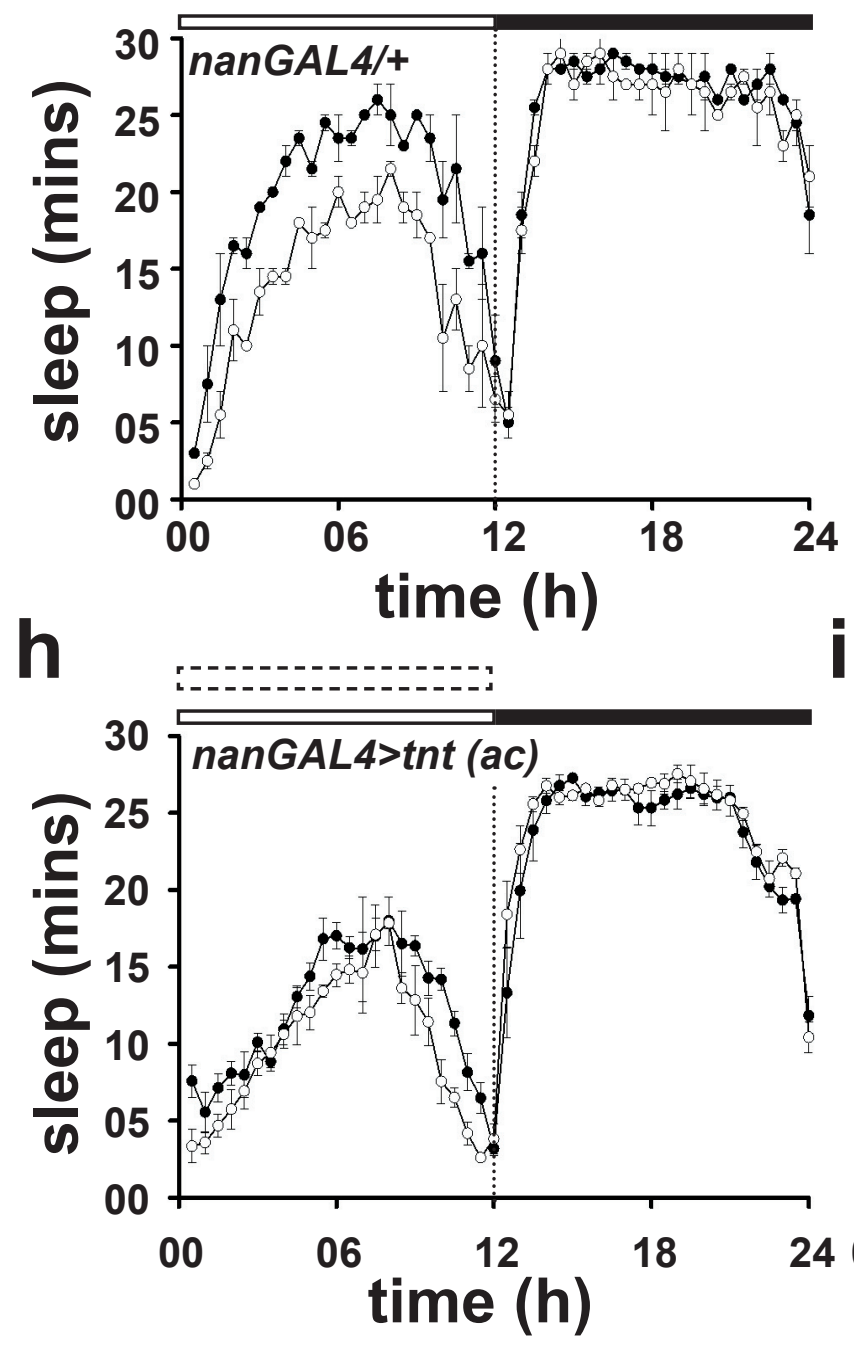

Control C

- OMD
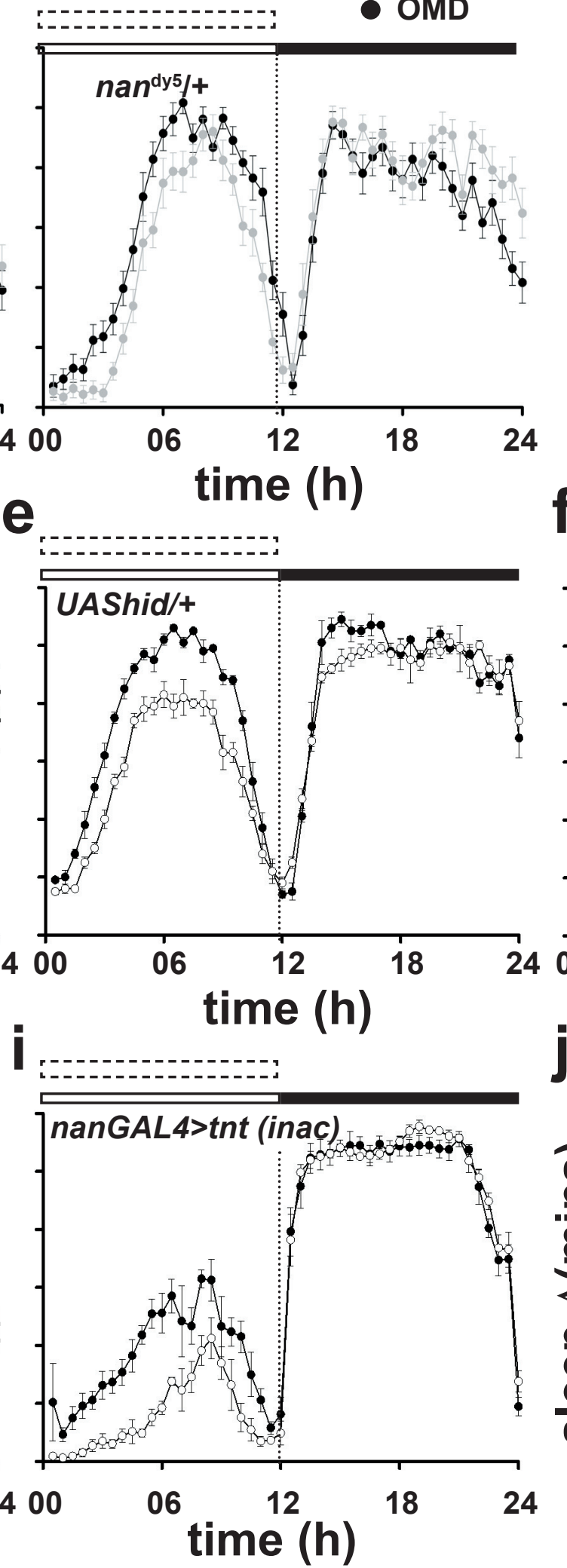

Figure 5

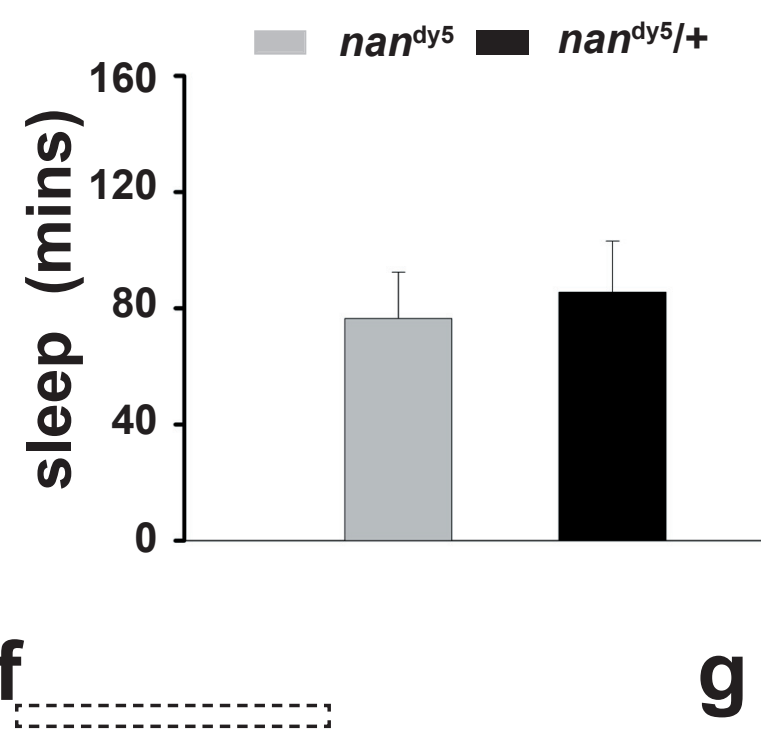

$\square$ nanGAL4/+

$\square$ UAShid/+

nanGAL4>hid
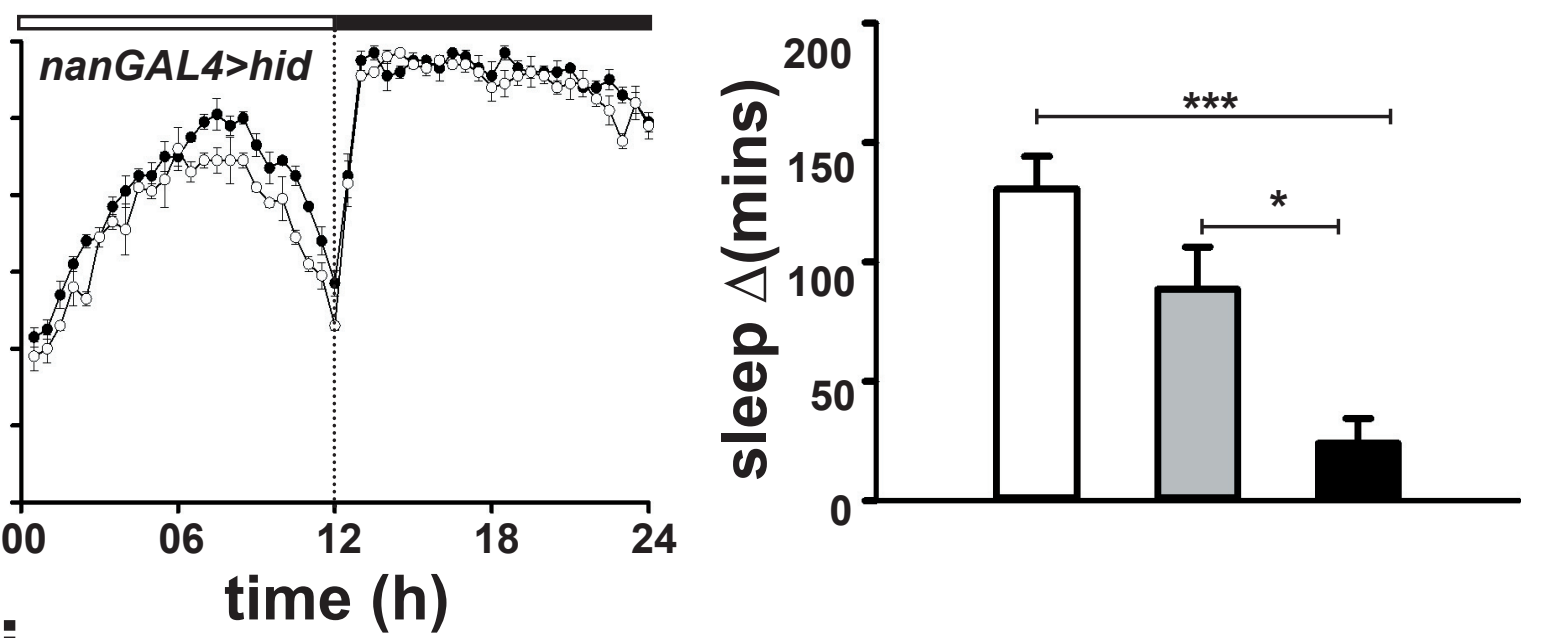

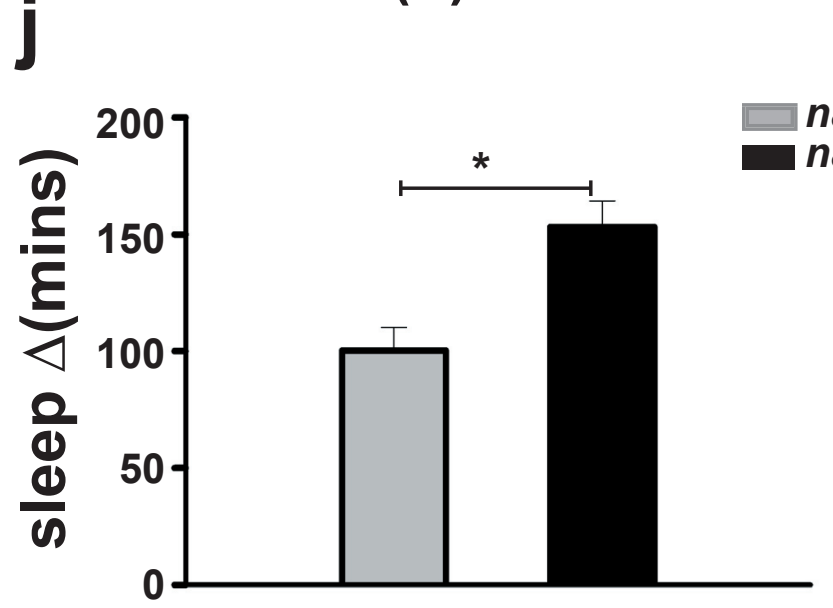

nanGAL4>tnt (ac)

nanGAL4>tnt (inac) 


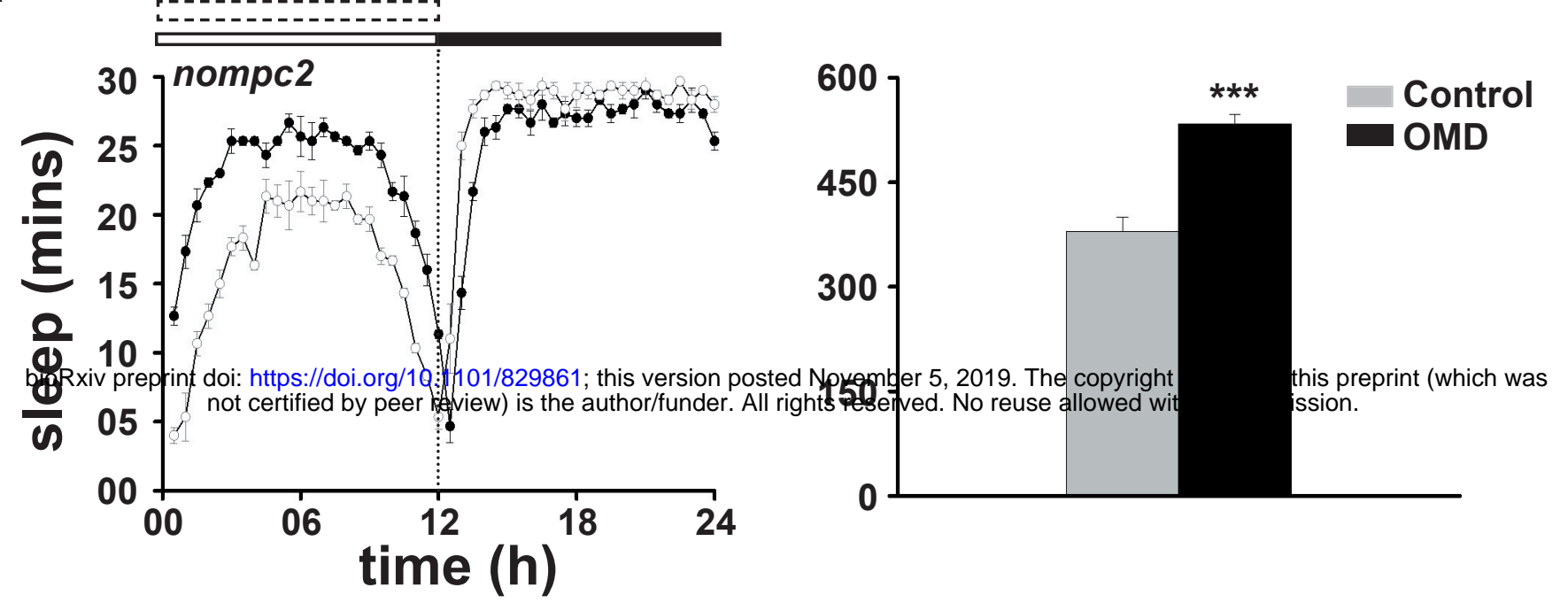

Figure 6

b ○ Control — OMD
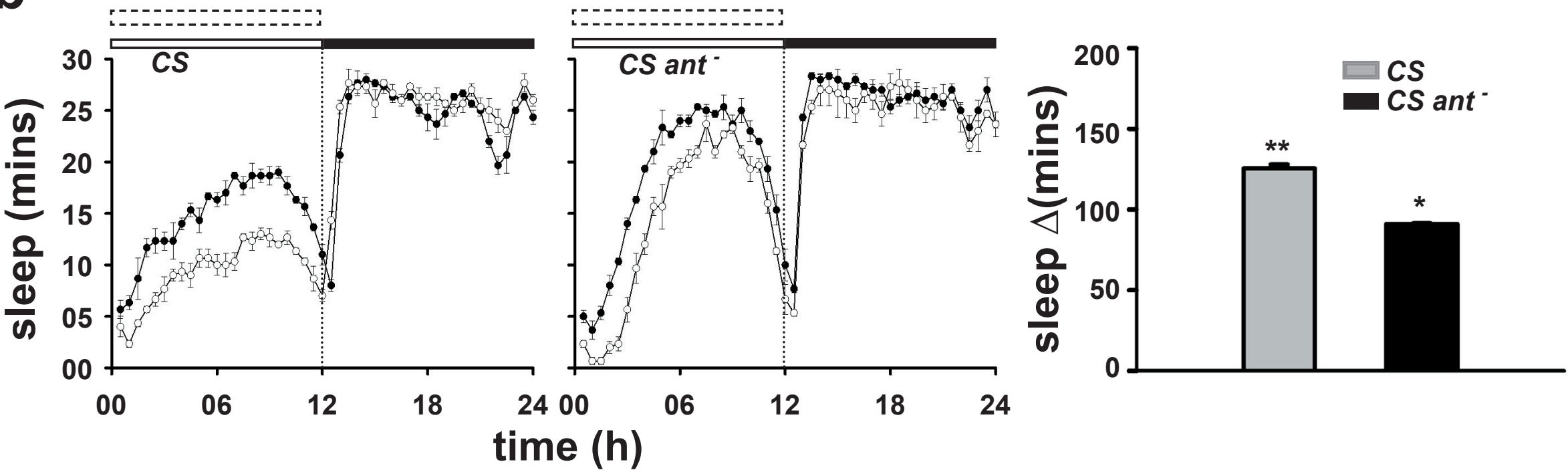

C

- Control O OMD

- Control

OMD
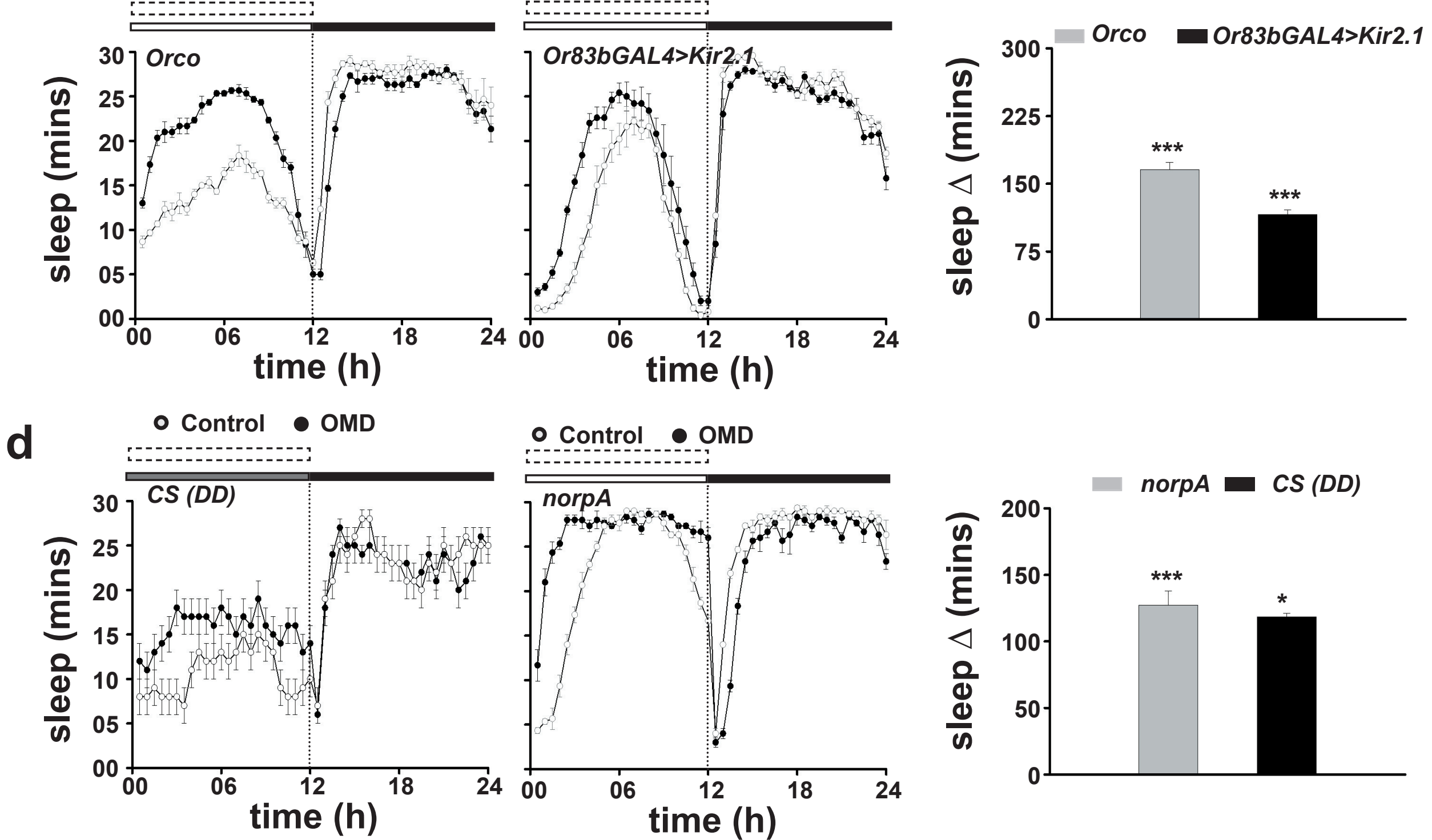
Figure 7

a

b
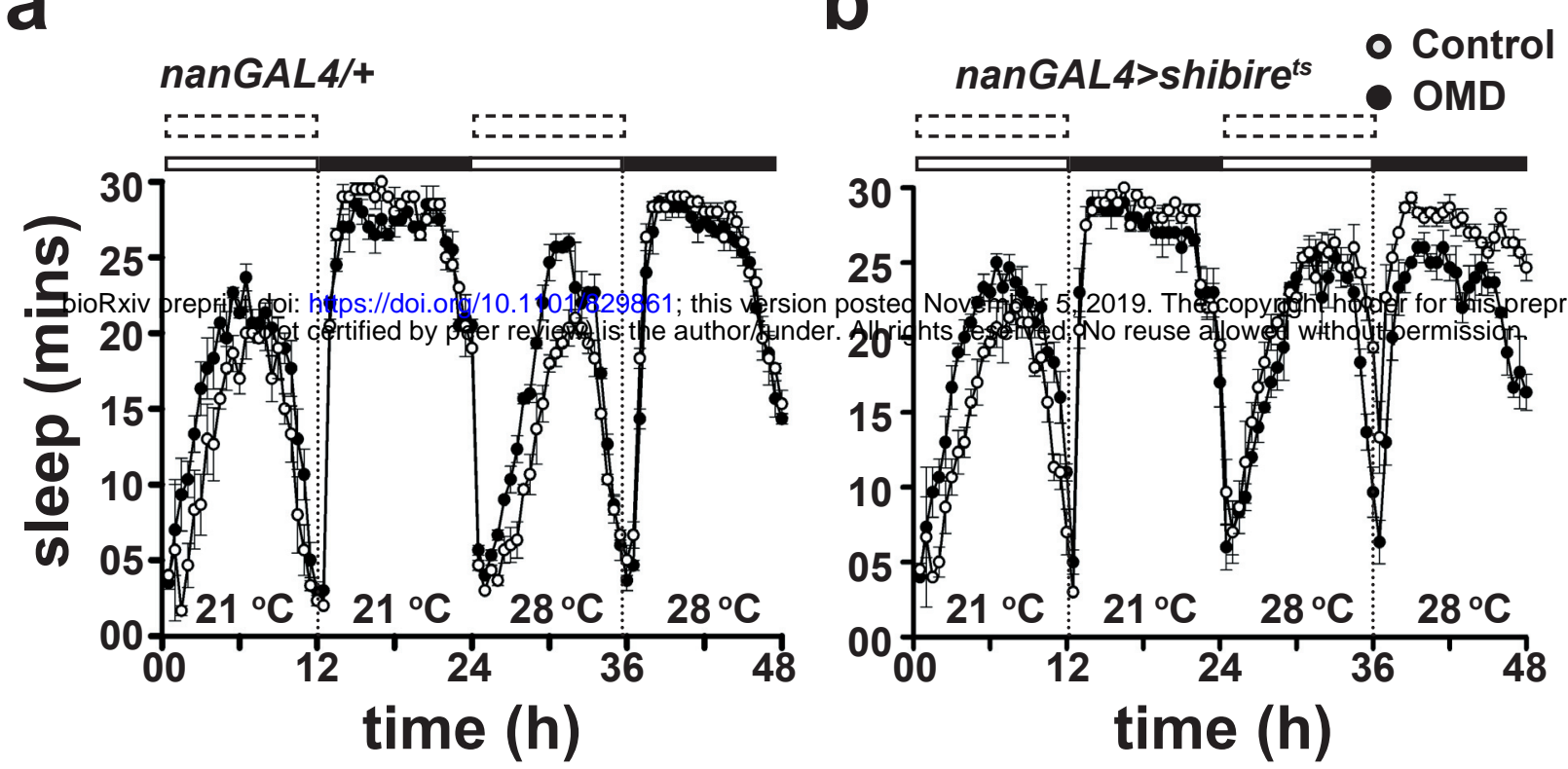

C

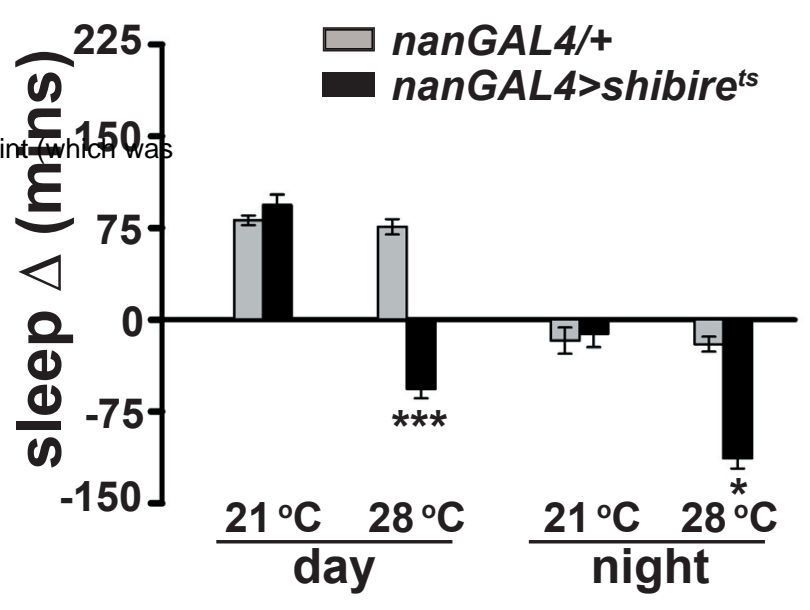

d

- nanGAL4>dTRPA1

e

- nanGAL4/+
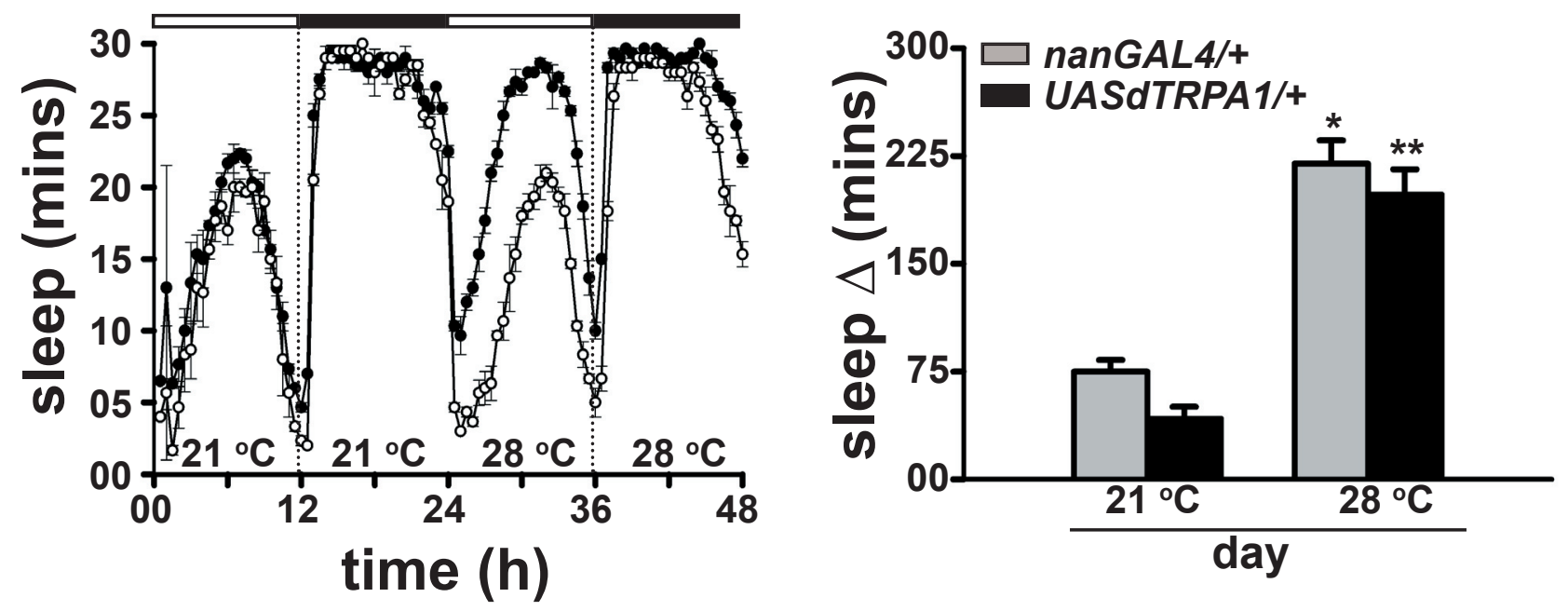

f $\quad \nabla \mathrm{M} \bullet \mathrm{MM} \bullet \mathrm{MF}$

g $\nabla \mathrm{M} \bullet \mathrm{MM} \bullet \mathrm{MF}$

h $=M-M M=M F$
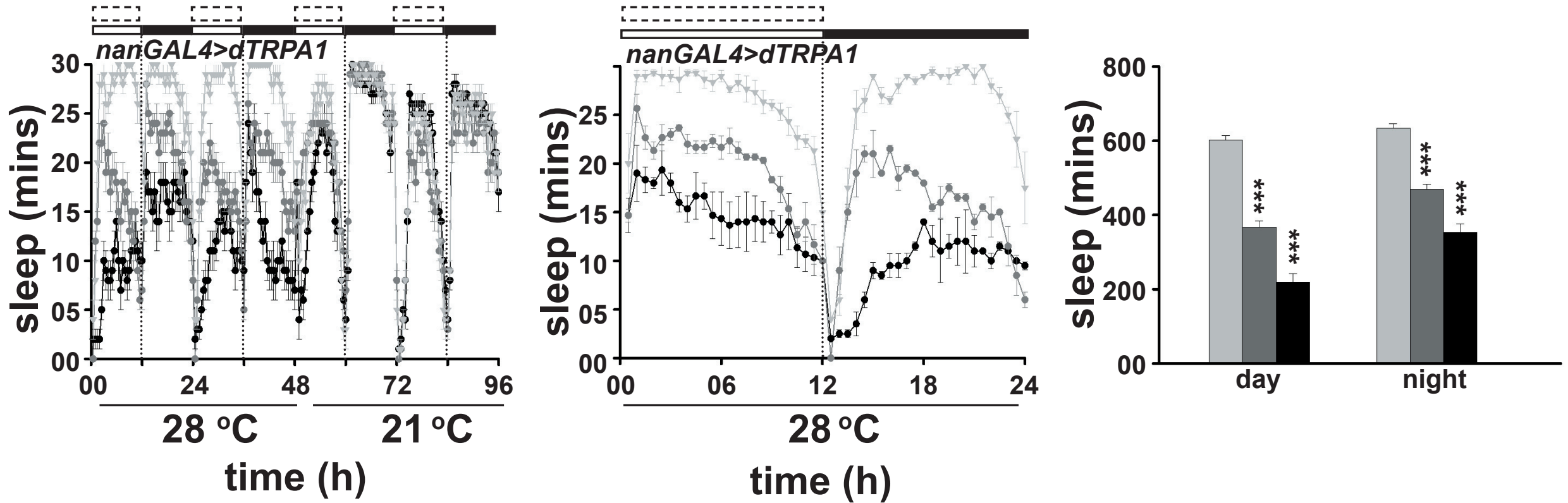
a

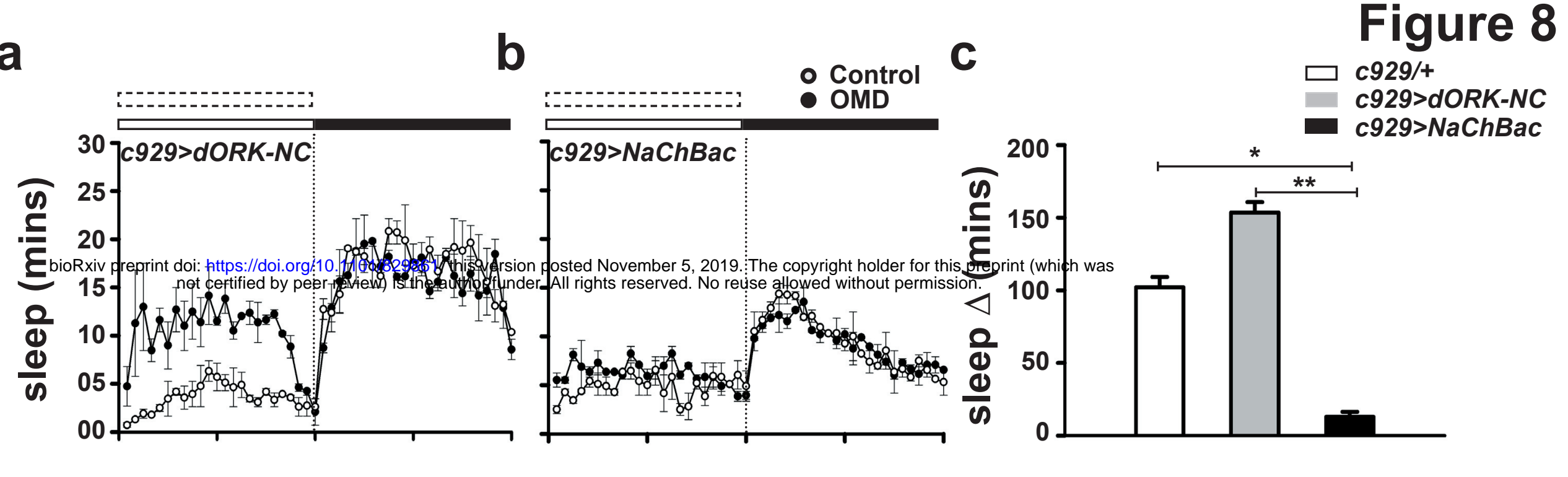

d
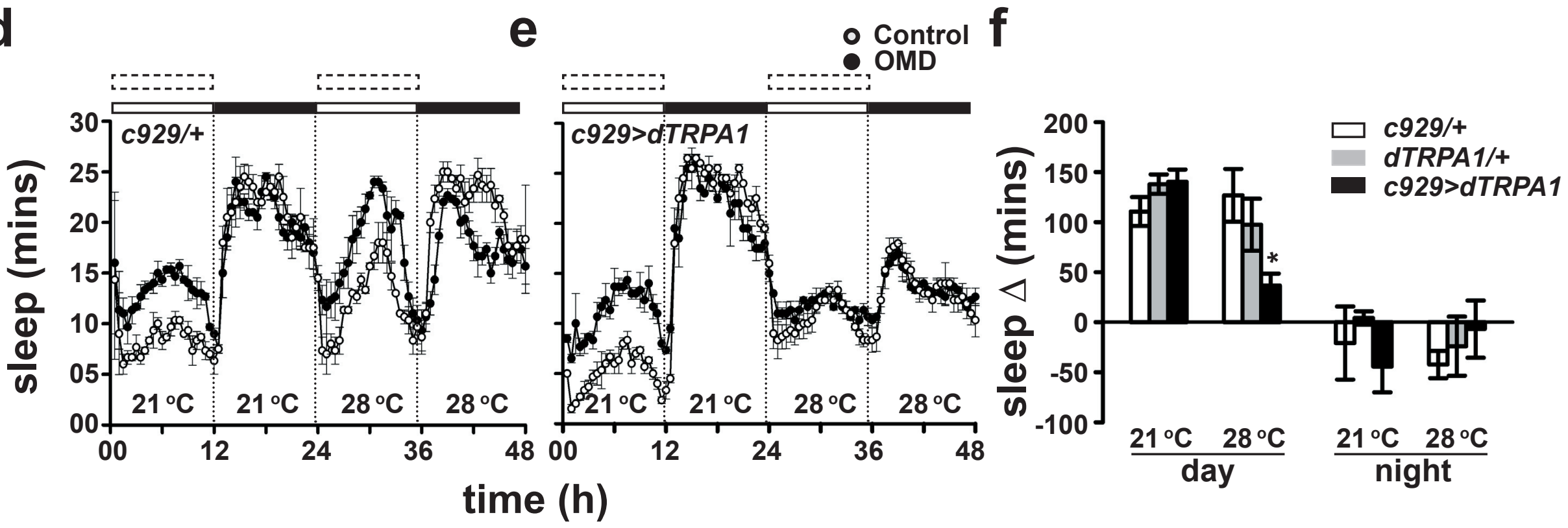